Alexander H. W. Schmitt

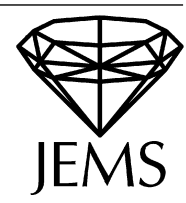

\title{
Singular principal $G$-bundles on nodal curves
}

Received August 5, 2004 and in revised form September 21, 2004

\begin{abstract}
In the present paper, we give a first general construction of compactified moduli spaces for semistable $G$-bundles on an irreducible complex projective curve $X$ with exactly one node, where $G$ is a semisimple linear algebraic group over the complex numbers.
\end{abstract}

Keywords. Principal bundle, nodal curve, generalized parabolic bundle, moduli space

\section{Introduction}

If $X$ is a smooth projective curve and $G$ is a reductive linear algebraic group, then one has the projective moduli space $\mathscr{M}_{G}^{\tau}$ for the (S-equivalence classes of) semistable principal $G$ bundles of fixed topological type $\tau \in \pi_{1}(G)$. The GIT-construction of this space is due to Ramanathan [14]. Later it was simplified and extended to higher dimensions by Gómez and Sols [10]. If $G$ is a semisimple group, there are alternative approaches by Balaji and Seshadri [1], and the author [16], [17]. As does the work of Gómez and Sols, the latter two yield Gieseker-type compactifications of the moduli spaces of stable principal bundles in higher dimensions. An important open problem, raised by M. S. Narasimhan, is to generalize this result to singular curves. The first case to understand will be the case where $X$ is an irreducible projective curve with exactly one node (which will be called for simplicity a nodal curve in what follows). If $G=\mathrm{GL}_{r}(\mathbb{C})$, one may work with vector bundles of rank $r$ rather than with principal $G$-bundles, and then the moduli space $\mathscr{U}(r, d)$ of semistable torsion free sheaves of rank $r$ and fixed degree $d$ is the generalization one is looking for. Similarly, if $G=\mathrm{O}(r)$ or $G=\mathrm{Sp}(r)$, then one may work with torsion free sheaves $\mathscr{E}$ together with a non-degenerate bilinear form $\beta: \mathscr{E} \otimes \mathscr{E} \rightarrow \mathscr{O}_{X}$, required to be symmetric in the case of $\mathrm{O}(r)$ and anti-symmetric in the case of $\mathrm{Sp}(r)$. For these objects, one has again a natural notion of semistability and the moduli spaces can be constructed [6], [3]. Surprisingly, however, the problem becomes difficult already for $G=$ $\mathrm{SL}_{r}(\mathbb{C})$. On a smooth curve, an $\mathrm{SL}_{r}(\mathbb{C})$-bundle is usually identified with a vector bundle $E$ with $\wedge^{\text {rkE}} E \cong \mathscr{O}_{X}$. This determinantal condition does not make sense for a nonlocally free torsion free sheaf $\mathscr{E}$ on a nodal curve. Nagaraj and Seshadri suggest replacing

A. H. W. Schmitt: FB6 Mathematik \& Informatik, Universität Duisburg-Essen, D-45117 Essen, Germany; e-mail: alexander.schmitt@uni-essen.de

Mathematics Subject Classification (2000): Primary 14H60; Secondary 14D20, 14L24 
the above condition by the condition that there be a non-zero homomorphism $\bigwedge^{\text {rk } \mathscr{E}} \mathscr{E} \rightarrow$ $\mathscr{O}_{X}$ which is an isomorphism outside the node of $X$ ([13, p. 136]). Sun has recently checked in [22] that this suggestion seems reasonable in the sense that this condition defines an irreducible closed subset $\mathscr{U}_{\mathrm{SL}_{r}(\mathbb{C})}$ in the moduli space of semistable torsion free sheaves of degree zero and rank $r$ on the curve $X$ which is compatible with degenerations. The latter statement means that, for a degeneration of a smooth curve $\widehat{X}$ to $X$, the moduli space of vector bundles of rank $r$ with trivial determinant on $\widehat{X}$ degenerates to a closed subscheme of $\mathscr{U}(r, 0)$ the support of which is exactly $\mathscr{U}_{\mathrm{SL}_{r}(\mathbb{C})}$. The open problem which remains is to give $\mathscr{U}_{\mathrm{SL}_{r}(\mathbb{C})}$ a modular interpretation.

\section{1}

Based on the notion of a singular principal bundle introduced by the author on smooth projective manifolds in [16] and extended in a certain way to a wide class of singular varieties (including nodal curves, see Section 5.2 for the discussion) by Bhosle [3], we propose to look at the following objects. Let $X$ be a nodal curve, $G$ a semisimple linear algebraic group, and $\varrho: G \rightarrow \operatorname{SL}(V)$ a faithful representation. An honest singular principal $G$-bundle on $X$ is a pair $(\mathscr{A}, \tau)$, consisting of a torsion free sheaf $\mathscr{A}$ on $X$ and a homomorphism $\tau: \operatorname{Sym}^{\star}(\mathscr{A} \otimes V)^{G} \rightarrow \mathscr{O}_{X}$ such that the image of $U:=X \backslash$ \{node\} under $\sigma$ is contained in $\mathscr{I}_{\operatorname{som}}\left(V \otimes \mathscr{O}_{X}, \mathscr{A}_{\mid U}^{\vee}\right) / G$. Here, $\sigma: X \rightarrow \mathscr{S p e c}\left(\operatorname{Sym}^{\star}(\mathscr{A} \otimes V)^{G}\right)=$ $\mathscr{H} o m\left(V \otimes \mathscr{O}_{X}, \mathscr{A}_{\mid U}^{\vee}\right) / / G$ is the section corresponding to $\tau$. Now, we can form the base change diagram

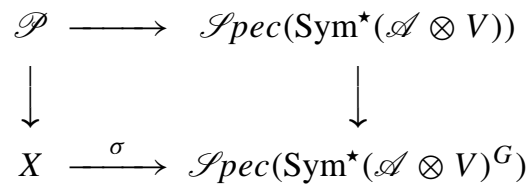

The condition on the image of $U$ guarantees that $\mathscr{P}(\mathscr{A}, \tau):=\mathscr{P}_{\mid U}$ is indeed a principal $G$-bundle. In order to formulate an appropriate semistability condition for honest singular $G$-bundles, we need some more notation. Suppose $\mathscr{A}$ is a torsion free sheaf on $X$; then a weighted filtration of $\mathscr{A}$ is a pair $\left(\mathscr{A}^{\bullet}, \underline{\alpha}\right)$, consisting of a filtration

$$
\mathscr{A}^{\bullet}: \quad 0 \subsetneq \mathscr{A}_{1} \subsetneq \cdots \subsetneq \mathscr{A}_{S} \subsetneq \mathscr{A}
$$

of $\mathscr{A}$ by saturated subsheaves and a tuple $\underline{\alpha}=\left(\alpha_{1}, \ldots, \alpha_{s}\right)$ of positive rational numbers. To a weighted filtration $\left(\mathscr{A}^{\bullet}, \underline{\alpha}\right)$, we associate the rational number

$$
L\left(\mathscr{A}^{\bullet}, \underline{\alpha}\right):=\sum_{i=1}^{s} \alpha_{i}\left(\operatorname{deg}(\mathscr{A}) \cdot \operatorname{rk} \mathscr{A}_{i}-\operatorname{deg}\left(\mathscr{A}_{i}\right) \cdot \operatorname{rk} \mathscr{A}\right) .
$$

Next, let $\lambda: \mathbb{C}^{\star} \rightarrow G$ be a one-parameter subgroup of $G$, and let

$$
Q_{G}(\lambda):=\left\{g \in G \mid \lim _{z \rightarrow \infty} \lambda(z) \cdot g \cdot \lambda(z)^{-1} \text { exists in } G\right\}
$$

be the associated parabolic subgroup. Recall also that $\lambda$ defines integers $\gamma_{1}<\cdots<\gamma_{s+1}$ and a decomposition 


$$
V:=\bigoplus_{i=1}^{s+1} V_{i}, \quad V_{i}:=\left\{v \in V \mid \lambda(z)(v)=z^{\gamma_{i}} \cdot v, \forall z \in \mathbb{C}^{\star}\right\}, i=1, \ldots, s+1,
$$

and thus the weighted flag $\left(V^{\bullet}, \underline{\alpha}\right)$ with

$$
V^{\bullet}: \quad 0 \subsetneq V_{1} \subsetneq V_{1} \oplus V_{2} \subsetneq \cdots \subsetneq V_{1} \oplus \cdots \oplus V_{s} \subsetneq V
$$

and

$$
\underline{\alpha}=\left(\alpha_{1}, \ldots, \alpha_{s}\right), \quad \alpha_{i}:=\frac{\gamma_{i+1}-\gamma_{i}}{\operatorname{dim} V}, \quad i=1, \ldots, s .
$$

We remark that $Q_{G}(\lambda)$ is the $G$-stabilizer of the flag $V^{\bullet}$. Then a reduction of $(\mathscr{A}, \tau)$ to $\lambda$ is a section $\beta: U \rightarrow \mathscr{P}(\mathscr{A}, \tau) / Q_{G}(\lambda)$. This defines a weighted filtration $\left(\mathscr{A}_{\beta}^{\bullet}, \underline{\alpha}_{\beta}\right)$ of $\mathscr{A}$ in the following manner: Set $\underline{\alpha}_{\beta}=\left(\alpha_{s}, \ldots, \alpha_{1}\right)$ where $\underline{\alpha}=\left(\alpha_{1}, \ldots, \alpha_{s}\right)$, and the filtration $\mathscr{A}_{\beta}^{\bullet}: 0 \subsetneq \mathscr{A}_{1} \subsetneq \cdots \subsetneq \mathscr{A}_{s} \subsetneq \mathscr{A}$ is obtained as follows: The section

$$
U \stackrel{\beta}{\rightarrow} \mathscr{P}(\mathscr{A}, \tau) / Q_{G}(\lambda) \hookrightarrow \mathscr{I}_{\operatorname{som}}\left(V \otimes \mathscr{O}_{U}, \mathscr{A}_{\mid U}^{\vee}\right) / Q_{\mathrm{GL}(V)}(\lambda)
$$

yields a filtration

$$
0 \subsetneq \mathscr{A}_{1}^{\prime} \subsetneq \cdots \subsetneq \mathscr{A}_{s}^{\prime} \subsetneq \mathscr{A}_{\mid U}^{\vee}
$$

of $\mathscr{A}_{\mid U}^{\vee}$ by subbundles with $\operatorname{rk}\left(\mathscr{A}_{i}^{\prime}\right)=\operatorname{dim}\left(V_{i}\right), i=1, \ldots, s$. This is because $Q_{\mathrm{GL}(V)}(\lambda)$ is the $\mathrm{GL}(V)$-stabilizer of the flag $V^{\bullet}$ and, thus, $\mathscr{I}_{\operatorname{som}}\left(V \otimes \mathscr{O}_{U}, \mathscr{A}_{\mid U}^{\vee}\right) / Q_{\mathrm{GL}(V)}(\lambda) \rightarrow U$ is the bundle of flags in the fibres of $\mathscr{A}_{\mid U}^{\vee}$ having the same dimensions as the flag $V^{\bullet}$. We define $\mathscr{A}_{i}^{\prime \prime}:=\operatorname{ker}\left(\mathscr{A}_{\mid U} \rightarrow \mathscr{A}_{s+1-i}^{\prime \vee}\right), i=1, \ldots, s$, so that we obtain a filtration

$$
0 \subsetneq \mathscr{A}_{1}^{\prime \prime} \subsetneq \cdots \subsetneq \mathscr{A}_{S}^{\prime \prime} \subsetneq \mathscr{A}_{\mid U}
$$

of $\mathscr{A}_{\mid U}$ by subbundles. Let $j: U \rightarrow X$ be the inclusion and define $\mathscr{A}_{i}$ as the saturation of $\mathscr{A} \cap j_{\star}\left(\mathscr{A}_{i}^{\prime \prime}\right), i=1, \ldots, s$. Now, an honest singular principal $G$-bundle is said to be (semi)stable if

$$
L\left(\mathscr{A}_{\beta}^{\bullet}, \underline{\alpha}_{\beta}\right)(\geq) 0
$$

for every reduction $\beta$ of $(\mathscr{A}, \tau)$ to a one-parameter subgroup of $G$.

1.2

Unfortunately, we are not able to give a direct construction of the moduli space of semistable honest singular $G$-bundles on the curve $X$. Instead, we use an idea inspired by the theory of generalized parabolic bundles of [2]. For this, let $N \in X$ be the node of $X, v: \widetilde{X} \rightarrow X$ the normalization, and $v^{-1}(N)=\left\{N_{1}, N_{2}\right\}$. Recall that a generalized parabolic bundle of rank $r$ is composed of 1) a vector bundle $A$ of rank $r$ on $\widetilde{X}$ and 2) an $r$-dimensional quotient $q: A_{N_{1}} \oplus A_{N_{2}} \rightarrow R$. The pair $(A, q)$ defines the torsion free sheaf $\mathscr{A}$ on $X$ as

$$
\mathscr{A}:=\operatorname{ker}\left(v_{\star} A \rightarrow v_{\star}\left(A_{N_{1}} \oplus A_{N_{2}}\right)=A_{N_{1}} \oplus A_{N_{2}}(\text { supported at } N) \stackrel{q}{\rightarrow} R\right) .
$$

First, look at a triple $(A, q, \widetilde{\tau})$, where $(A, q)$ is a generalized parabolic bundle as above, and $\tilde{\tau}: \operatorname{Sym}^{\star}(A \otimes V)^{G} \rightarrow \mathscr{O}_{\tilde{X}}$ is a homomorphism of $\mathscr{O}_{\widetilde{X}}$-algebras. Then we define the 
pushforward $(\mathscr{A}, \tau):=v_{\star}(A, q, \widetilde{\tau})$ as follows: The torsion free sheaf $\mathscr{A}$ is associated to $(A, q)$ as explained before. In order to define the homomorphism $\tau: \operatorname{Sym}^{\star}(\mathscr{A} \otimes V)^{G} \rightarrow$ $\mathscr{O}_{X}$ of $\mathscr{O}_{X}$-algebras, we first observe that the inclusion $\mathscr{A} \subset v_{\star}(A)$ corresponds to a homomorphism $v^{\star}(\mathscr{A}) \rightarrow A$. This yields a homomorphism

$$
\tau^{\prime}: v^{\star}\left(\operatorname{Sym}^{\star}(\mathscr{A} \otimes V)^{G}\right) \cong \operatorname{Sym}^{\star}\left(v^{\star}(\mathscr{A}) \otimes V\right)^{G} \rightarrow \operatorname{Sym}^{\star}(A \otimes V)^{G} \stackrel{\tau}{\rightarrow} \mathscr{O}_{\widetilde{X}}
$$

of $\mathscr{O}_{\widetilde{X}}$-algebras. The isomorphism results from general base change properties (Section 2 of [16]), and the second homomorphism comes from the natural map $v^{\star}(\mathscr{A}) \rightarrow A$. Now, $\tau^{\prime}$ in turn provides us with the map

$$
\tau: \operatorname{Sym}^{\star}(\mathscr{A} \otimes V)^{G} \rightarrow v_{\star} \nu^{\star}\left(\operatorname{Sym}^{\star}(\mathscr{A} \otimes V)^{G}\right) \stackrel{\nu_{\star}\left(\tau^{\prime}\right)}{\rightarrow} v_{\star}\left(\mathscr{O}_{\widetilde{X}}\right) .
$$

This is a homomorphism of $\mathscr{O}_{X}$-algebras. To see this, one has to verify that the first map is a homomorphism of $\mathscr{O}_{X}$-algebras. The latter reduces to the corresponding property of the symmetric algebra $\operatorname{Sym}^{\star}(\mathscr{A} \otimes V)$, which, finally, is easily checked.

Now, we define a descending principal $G$-bundle $($ on $\widetilde{X})$ to be a triple $(A, q, \widetilde{\tau})$ as before, such that the following requirements are met:

- The pair $(A, \widetilde{\tau})$ defines by means of base change (see Diagram (1p) a principal $G$ bundle $\mathscr{P}(A, \widetilde{\tau})$ on $\widetilde{X}$. This is equivalent to the fact that the associated section $\widetilde{\sigma}: \widetilde{X} \rightarrow$ $\mathscr{H} o m\left(V \otimes \mathscr{O}_{\tilde{X}}, A^{\vee}\right) / / G$ given by $\tilde{\tau}$ factorizes over the open subscheme $\mathscr{I}_{\operatorname{som}}(V \otimes$ $\left.\mathscr{O}_{\widetilde{X}}, A^{\vee}\right) / G$. Note that this implies that $\operatorname{det}(A) \cong \mathscr{O}_{\widetilde{X}}$.

- The image of the homomorphism $\tau$ from the pair $(\mathscr{A}, \tau)=v_{\star}(A, q, \widetilde{\tau})$ lies in the subalgebra $\mathscr{O}_{X}$.

Remark 1.2.1. (i) By definition, the pair $\left(\mathscr{A}, \tau: \operatorname{Sym}^{\star}(\mathscr{A} \otimes V)^{G} \rightarrow \mathscr{O}_{X}\right)$ is an honest singular $G$-bundle on the nodal curve $X$. Thus, in analogy with Bhosle's theory of generalized parabolic bundles, we describe honest singular $G$-bundles on the singular curve $X$ by principal $G$-bundles on the smooth curve $\widetilde{X}$, satisfying some descent conditions.

(ii) A principal $G$-bundle $P$ on $X$ defines a descending $G$-bundle on $\widetilde{X}$. For this, let $\mathscr{E}$ be the vector bundle with fibre $V$ associated with $P$ by means of the representation $\varrho$, and set $\mathscr{A}:=\mathscr{E}^{\vee}$. We have the natural inclusion $P \subset \mathscr{I}_{\operatorname{som}}\left(V \otimes \mathscr{O}_{X}, \mathscr{A}^{\vee}\right)$. Taking the $G$-quotient, we find the section $\sigma: X \rightarrow \mathscr{I}_{\operatorname{som}}\left(V \otimes \mathscr{O}_{X}, \mathscr{A}^{\vee}\right) / G$ which corresponds to a homomorphism $\tau: \operatorname{Sym}^{\star}(\mathscr{A} \otimes V)^{G} \rightarrow \mathscr{O}_{X}$ of $\mathscr{O}_{X}$-algebras. Then we set $A:=$

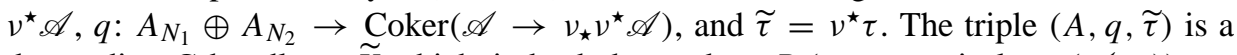
descending $G$-bundle on $\widetilde{X}$ which, indeed, descends to $P$ (more precisely to $(\mathscr{A}, \tau)$ ).

1.3

Next, we have to define the semistability concept for descending principal $G$-bundles. This concept will depend on a parameter $\alpha \in(0,1) \cap \mathbb{Q}$. Fix such a parameter $\alpha$, and let $(A, q)$ be a generalized parabolic bundle as above. For any subbundle $B$ of $A$, we define the $\alpha$-parabolic degree as

$$
\alpha-\operatorname{pardeg}(B):=\operatorname{deg}(B)-\alpha \cdot \operatorname{dim} q\left(B_{N_{1}} \oplus B_{N_{2}}\right) .
$$


Now, let $(A, q, \tilde{\tau})$ be a descending $G$-bundle on the curve $\tilde{X}$. As before, we can speak of a weighted filtration $\left(A^{\bullet}, \underline{\alpha}\right)$ of $A$ (where "saturated subsheaf" = "subbundle", because $\widetilde{X}$ is smooth). To such a weighted filtration $\left(A^{\bullet}, \underline{\alpha}\right)$, we assign the rational number

$$
P_{\alpha}\left(A^{\bullet}, \underline{\alpha}\right):=\sum_{i=1}^{s} \alpha_{i}\left(\alpha-\operatorname{pardeg}(A) \cdot \operatorname{rk} A_{i}-\alpha-\operatorname{pardeg}\left(A_{i}\right) \cdot \operatorname{rk} A\right) .
$$

In analogy to the definitions above, a reduction of the descending $G$-bundle $(A, q, \tilde{\tau})$ to the one-parameter subgroup $\lambda: \mathbb{C}^{\star} \rightarrow G$ is a section $\beta: \tilde{X} \rightarrow \mathscr{P}(A, q, \tilde{\tau}) / Q_{G}(\lambda)$. Such a reduction $\beta$ gives rise to a weighted filtration $\left(A_{\beta}^{\bullet}, \underline{\alpha}_{\beta}\right)$ of $A$ by a procedure similar to the one used in the case of honest singular principal bundles on the nodal curve $X$. Finally, we call $(A, q, \widetilde{\tau}) \alpha-($ semi $)$ stable if the condition

$$
P_{\alpha}\left(\mathscr{A}_{\beta}^{\bullet}, \underline{\alpha}_{\beta}\right)(\geq) 0
$$

holds for any reduction $\beta$ of $(A, q, \widetilde{\tau})$ to a one-parameter subgroup of $G$.

Below, we will explain what a family of descending $G$-bundles parameterized by $a$ scheme $S$ is. When one has this notion at hand, one may define moduli functors

$$
\begin{aligned}
\underline{\mathrm{M}}(\varrho)^{\alpha-(\mathrm{s}) \mathrm{s}}: \underline{\mathrm{Sch}}_{\mathbb{C}} & \rightarrow \underline{\mathrm{Set}}, \\
S & \mapsto\left\{\begin{array}{l}
\text { equivalence classes of families of } \\
\alpha \text {-(semi)stable descending } G \text {-bundles }
\end{array}\right\} .
\end{aligned}
$$

We then have:

Main Theorem. There exist a projective scheme $\mathscr{M}(\varrho)^{\alpha-s s}$ and an open subscheme $\mathscr{M}(\varrho)^{\alpha-s} \subset \mathscr{M}(\varrho)^{\alpha \text {-ss }}$ as well as natural transformations of functors

$$
\vartheta^{\alpha-(\mathrm{s}) \mathrm{s}}: \underline{\mathrm{M}}(\varrho)^{\alpha-(\mathrm{s}) \mathrm{s}} \rightarrow h_{\mathscr{M}}(\varrho)^{\alpha-(\mathrm{s}) \mathrm{s}}
$$

with the following properties:

1. For every scheme $\mathscr{N}$ and every natural transformation $\vartheta^{\prime}: \mathscr{M}(\varrho)^{\alpha-\mathrm{ss}} \rightarrow h_{\mathscr{N}}$, there is a unique morphism $\psi: \mathscr{M}(\varrho)^{\alpha \text {-ss }} \rightarrow \mathscr{N}$ with $\vartheta^{\prime}=h(\psi) \circ \vartheta^{\alpha \text {-ss }}$.

2. The scheme $\mathscr{M}(\varrho)^{\alpha-\mathrm{s}}$ is a coarse moduli space for the functor $\underline{\mathrm{M}}(\varrho)^{\alpha-\mathrm{s}}$.

Regarding the relation of the notion of $\alpha$-(semi)stability to our notion of (semi)stability for honest singular $G$-bundles on nodal curves, we note

Proposition. There is a value $\alpha_{1} \in(0,1) \cap \mathbb{Q}$ such that, for every $\alpha>\alpha_{1}$ and every descending $G$-bundle $(A, q, \widetilde{\tau})$ on $\widetilde{X}$ with associated honest singular $G$-bundle $(\mathscr{A}, \tau)$ on $X$, the following conclusions hold true:

(i) If $(\mathscr{A}, \tau)$ is stable, then $(A, q, \tilde{\tau})$ is $\alpha$-stable.

(ii) If $(A, q, \widetilde{\tau})$ is $\alpha$-semistable, then $(\mathscr{A}, \tau)$ is semistable.

Note that we may apply our results also to the case $G=\mathrm{SL}_{r}(\mathbb{C})$ and $\varrho: \mathrm{SL}_{r}(\mathbb{C}) \subset$ $\mathrm{GL}_{r}(\mathbb{C})$. In this case, the moduli space $\mathscr{M}(\varrho)^{\alpha \text {-ss }}$ maps onto the closed subset $\mathscr{U}_{\mathrm{SL}_{r}(\mathbb{C})}$

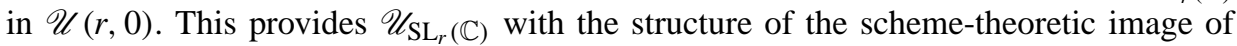
$\mathscr{M}(\varrho)^{\alpha \text {-ss }}$ and seems to be the most natural scheme structure one can put on $\mathscr{U}_{\mathrm{SL}_{r}(\mathbb{C})}$ at the moment. A similar strategy will be used to construct natural subschemes of Bhosle's "big" moduli spaces for singular principal $G$-bundles for an arbitrary semisimple group $G$. 
1.4

If $X$ has arithmetic genus one (i.e., $X$ is a nodal elliptic curve) and $G$ is simple and simply connected, there is another conceptual approach to the problem by Friedman and Morgan [8]. They construct a projective moduli space $\mathscr{M}_{\text {FM }}$ parameterizing certain $G$-bundles on $X$. (It seems to be specific to the case $p_{a}(X)=1$ that one can build a compact moduli space from principal $G$-bundles on $X$ only, i.e., without singular objects.) This moduli space is compatible with degenerations in the following sense: If $(\pi: \mathfrak{X} \rightarrow B, \sigma: B \rightarrow \mathfrak{X}$ ) consists of a flat proper morphism $\pi$ the fibres of which are either smooth or nodal elliptic curves and a section $\sigma$ which meets the fibres only in smooth points, then there is a relative moduli space $\mathscr{M}_{\mathfrak{X} / B} \rightarrow B$ such that the fibre over a point $b$ is the usual moduli space of semistable $G$-bundles on $\pi^{-1}(b)$ if that curve is smooth, and $\mathscr{M}_{\mathrm{FM}}$ if $\pi^{-1}(b)$ is a nodal curve. Let $B^{0}$ be the open part where the fibres of $\pi$ are smooth elliptic curves. Then the relative compactification $\mathscr{M}_{\mathfrak{X} / B}$ of $\mathscr{M}_{\pi^{-1}\left(B^{0}\right) / B^{0}}$ has certain uniqueness properties (see Section 5 of [8]).

The moduli space $\mathscr{M}_{\mathrm{FM}}$ is obtained by generalizing the "parabolic construction" introduced in [7] for smooth elliptic curves. In this construction, one fixes a system $\Delta$ of simple roots and a special root $\alpha \in \Delta$ (that is, any root if $G$ is of type $A_{n}$, and the unique long root such that the Dynkin diagram is a union of graphs of type $A$ with $\alpha$ as an end, meeting each other in $\alpha$ only, otherwise). This provides the maximal parabolic subgroup $P_{\alpha}$. The $G$-bundles which are classified by $\mathscr{M}_{\mathrm{FM}}$ are obtained from $P_{\alpha}$-bundles by means of extending the structure group. The $P_{\alpha}$-bundles, in turn, can be described by certain vector bundles and non-abelian cohomology groups. Although the parabolic construction gives very precise information on the $G$-bundles thus obtained, it seems hard to characterize them by semistability conditions. Nevertheless, the moduli space $\mathscr{M}_{\text {FM }}$ contains a dense affine open part $\mathscr{M}^{0}$ which parameterizes the $G$-bundles $P$ on $X$ enjoying the following equivalent properties:

- The pullback of $P$ to the normalization $\tilde{X}$ is trivial.

- For every representation $\varphi: G \rightarrow \operatorname{GL}(W)$, the vector bundle $\varphi_{*}(P)$ is semistable.

We claim that $\mathscr{M}^{0}$ also forms an open subset of our moduli spaces $\mathscr{M}(\varrho)^{\alpha \text {-ss }}$, for any representation $\varrho$ and any stability parameter $\alpha \in(0,1) \cap \mathbb{Q}$. To see this, let $P$ be a principal $G$-bundle on $X$ and consider the associated descending $G$-bundle $(A, \tilde{\tau}, q)$ (Remark 1.2.1(ii)). Note that $\Gamma:=\operatorname{ker}(q)$ may be viewed as the graph of the natural isomorphism $\varphi: A_{N_{1}} \rightarrow A_{N_{2}}$. For every subbundle $0 \subsetneq B \subsetneq A$, it follows that

$$
\operatorname{dim}\left(\left(B_{N_{1}} \oplus B_{N_{2}}\right) \cap \Gamma\right)=\operatorname{dim}\left(B_{N_{2}} \cap \varphi\left(B_{N_{1}}\right)\right) \leq \operatorname{rk}(B),
$$

i.e.,

$$
\operatorname{dim} q\left(B_{N_{1}} \oplus B_{N_{2}}\right) \geq \operatorname{rk}(B) .
$$

Thus, for any weighted filtration $\left(A^{\bullet}, \underline{\alpha}\right)$ of $A$, we obtain

$$
P\left(A^{\bullet}, \underline{\alpha}\right) \geq \sum_{i=1}^{s} \alpha_{i}\left(\operatorname{deg}(A) \cdot \operatorname{rk} A_{i}-\operatorname{deg}\left(A_{i}\right) \cdot \operatorname{rk} A\right) \geq 0 .
$$

The latter inequality results from the fact that $A$ is semistable. Thus, $\mathscr{M}(\varrho)^{\alpha-\text { ss }}$ and $\mathscr{M}_{\mathrm{FM}}$ are isomorphic over $\mathscr{M}^{0}$. 
At the moment, it seems to be difficult to obtain more precise information about the relationship between $\mathscr{M}(\varrho)^{\alpha \text {-ss }}$ and $\mathscr{M}_{\mathrm{FM}}$, because (a) the objects in $\mathscr{M}_{\mathrm{FM}}$ are not characterized by a semistability condition and (b) our moduli spaces $\mathscr{M}(\varrho)^{\alpha \text {-ss }}$ are probably not compatible with degenerations (so that the uniqueness property mentioned above cannot be applied). Hopefully, more precise knowledge on the problem raised in Section 5.3 will shed light on this. Finally, it would be interesting to know whether the description of the category of coherent sheaves on $X$ given by Burban and Drozd in [5] might be used and generalized to get a description of all singular $G$-bundles on $X$.

\section{Conventions}

We work over the field of complex numbers. A scheme will be a scheme of finite type over $\mathbb{C}$. For a vector bundle $\mathscr{E}$ over a scheme $X$, we set $\mathbb{P}(\mathscr{E}):=\operatorname{Proj}\left(\operatorname{Sym}^{\star}(\mathscr{E})\right)$, i.e., $\mathbb{P}(\mathscr{E})$ is the projective bundle of hyperplanes in the fibres of $\mathscr{E}$.

\section{Preliminaries}

\subsection{Base change properties of affine morphisms}

Suppose that $f: X \rightarrow Y$ is an affine morphism, and let $h: Z \rightarrow Y$ be an arbitrary morphism. We form the base change diagram

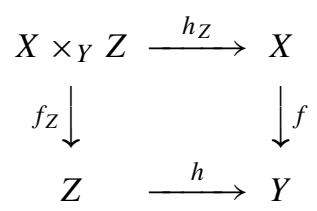

The morphism $f_{Z}$ is again affine, and one has

Proposition 2.1.1. For any coherent sheaf $\mathscr{E}$ on $X$, the natural base change homomorphism

$$
h^{\star} f_{\star}(\mathscr{E}) \rightarrow f_{Z \star} h_{Z}^{\star}(\mathscr{E})
$$

is an isomorphism.

Proof. This can be found in [11, p. 12, Corollary (1.5.2)].

For any morphism $\varphi: T \rightarrow S$ between schemes and any $\mathscr{O}_{S}$-module $\mathscr{A}$, there is the "natural homomorphism" $\mathscr{A} \rightarrow \varphi_{\star} \varphi^{\star}(\mathscr{A})$.

Lemma 2.1.2. In the situation of Proposition 2.1.1 let $\mathscr{E}_{Y}$ be an $\mathscr{O}_{Y}$-module. Then the homomorphism

$$
h^{\star} \mathscr{E}_{Y} \stackrel{h^{\star}(\text { natural })}{\longrightarrow} h^{\star} f_{\star} f^{\star} \mathscr{E}_{Y} \stackrel{2.1 .1}{\cong} f_{Z \star} h_{Z}^{\star} f^{\star} \mathscr{E}_{Y}=f_{Z \star} f_{Z}^{\star} h^{\star} \mathscr{E} Y
$$

is the natural homomorphism for $\mathscr{A}:=h^{\star} \mathscr{E}_{Y}$ and $\varphi:=f_{Z}$.

Proof. This is easily checked in the setting of modules over rings. 


\subsection{Zero loci of sheaf homomorphisms}

Proposition 2.2.1. Let $X$ be a projective variety, $S$ a Noetherian scheme, $\mathscr{A}_{S}^{1}$ and $\mathscr{A}_{S}^{2}$ coherent sheaves on $S \times X$, and $\varphi_{S}: \mathscr{A}_{S}^{1} \rightarrow \mathscr{A}_{S}^{2}$ a homomorphism. If $\mathscr{A}_{S}^{2}$ is flat over $S$, then there is a closed subscheme $\mathfrak{Z} \subset S$ enjoying the following universal property: A morphism $h: T \rightarrow S$ factorizes over $\mathfrak{Z}$ if and only if $\left(h \times \mathrm{id}_{X}\right)^{\star} \varphi_{S}$ is trivial. In particular, $a$ point $s \in S$ lies in $\mathfrak{Z}$ if and only if $\varphi_{S \mid\{s\} \times X} \equiv 0$.

Proof. The author learned this result from [9, Lemma 3.1]. A proof is also given in [16. Proposition 2.1].

\subsection{Generalized parabolic bundles}

We summarize the main results of the paper [2]. It serves also as a model for the kind of results we wish to obtain for principal bundles. For this, we use the notation of the introduction. Recall that we work on a fixed nodal curve $X$ and that $\nu: \widetilde{X} \rightarrow X$ is the normalization map. As before, $N \in X$ is the singular point, and $v^{-1}(N)=\left\{N_{1}, N_{2}\right\}$.

In the introduction, we explained the notion of a generalized parabolic bundle. Now, we will introduce the notion of a family of such objects. For doing so, we use the following shorthand notations: If $S$ is the parameter scheme, then we write $S_{N_{1}, N_{2}}$ for the subscheme $S \times\left\{N_{1}, N_{2}\right\}$ of $S \times \widetilde{X}$ and $S_{N}$ for the subscheme $S \times\{N\}$ of $S \times X$. Then a family of generalized parabolic bundles parameterized by $S$ is a pair $\left(A_{S}, q_{S}\right)$ which consists of a vector bundle $A_{S}$ on $S \times \widetilde{X}$ and a quotient map $q_{S}: \bar{\pi}_{S \star}\left(A_{S \mid S_{N_{1}, N_{2}}}\right) \rightarrow R_{S}$ onto a vector bundle of rank $r$ on $S_{N}$. Here, $r$ is the rank of $A_{S}$ and $\bar{\pi}_{S}$ denotes the projection morphism $S_{N_{1}, N_{2}} \rightarrow S_{N}$. Two such families $\left(A_{S}^{1}, q_{S}^{1}\right)$ and $\left(A_{S}^{2}, q_{S}^{2}\right)$ are called equivalent if there is an isomorphism $\psi_{S}: A_{S}^{1} \rightarrow A_{S}^{2}$ such that

$$
q_{S}^{1}=q_{S}^{2} \circ \bar{\pi}_{S \star}\left(\psi_{S \mid S_{N_{1}, N_{2}}}\right) .
$$

Next, suppose $T$ is another scheme, $f: T \rightarrow S$ is a morphism, and $\left(A_{S}, q_{S}\right)$ is a family of generalized parabolic bundles parameterized by $S$. The pullback $\left(A_{T}, q_{T}\right)$ of the family $\left(A_{S}, q_{S}\right)$ via $f$ is obtained as follows: We start with the following diagram in order to introduce the necessary notation (where the maps $h, \widetilde{h}$, and $\widetilde{h}$ are derived from $f$, e.g., $\left.h=f \times \operatorname{id}_{X}\right)$ :

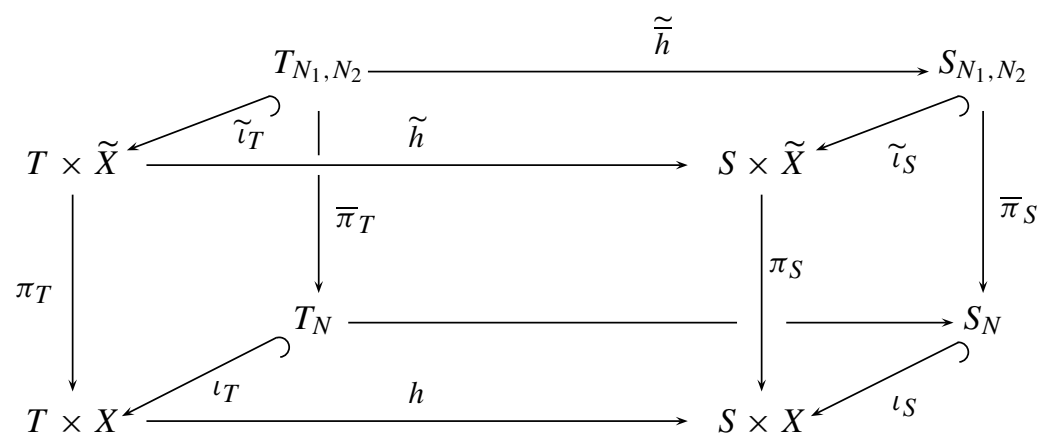


The given datum is the quotient $q_{S}: \pi_{S_{\star}} \widetilde{l}_{S \star} \widetilde{l}_{S}^{\star} A_{S} \rightarrow R_{S}$. This provides us, by means of pullback, with the quotient

$$
h^{\star} q_{S}: h^{\star} \pi_{S \star} \widetilde{l}_{\star} \widetilde{l}_{S}^{\star} A_{S} \rightarrow h^{\star} R_{S}=: R_{T}
$$

on the scheme $T_{N}$. A repeated application of Proposition 2.1.1 yields the following identifications:

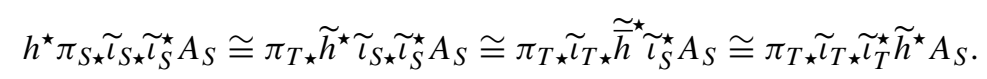

If we set $A_{T}:=\widetilde{h}^{\star} A_{S}$, then the above identifications turn $h^{\star} q_{S}$ into a quotient $q_{T}$ : $\tilde{\pi}_{T \star}\left(A_{T \mid T_{N_{1}, N_{2}}}\right) \rightarrow R_{T}$ such that the following diagram commutes:

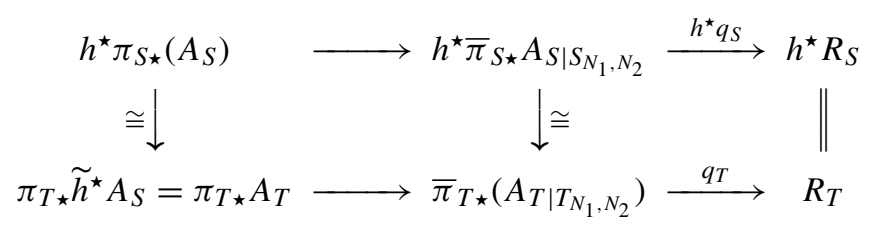

The associated family of torsion free sheaves on $S \times X$. Let $S$ be a scheme and $\left(A_{S}, q_{S}\right)$ a family of generalized parabolic bundles parameterized by $S$. We define the associated family of torsion free sheaves on $S \times X$ as

$$
\mathscr{A}_{S}:=\operatorname{ker}\left(\pi_{S \star}\left(A_{S}\right) \rightarrow \bar{\pi}_{S_{\star}}\left(A_{S \mid S_{N_{1}, N_{2}}}\right) \stackrel{q_{S}}{\rightarrow} R_{S}\right) .
$$

Since the map $\pi_{S_{\star}}\left(A_{S}\right) \rightarrow R_{S}$ is surjective and both $\pi_{S_{\star}}\left(A_{S}\right)$ and $R_{S}$ are $S$-flat, the family $\mathscr{A}_{S}$ is also $S$-flat.

Lemma 2.3.1. Let $\left(A_{S}, q_{S}\right)$ be a family of generalized parabolic bundles parameterized by $S, h: T \rightarrow S$ a homomorphism, and $\left(A_{T}, q_{T}\right)$ the pullback of $\left(A_{S}, q_{S}\right)$ by $h$. This yields the families $\mathscr{A}_{T}$ and $\mathscr{A}_{S}$ on $T \times X$ and $S \times X$ associated to $\left(A_{T}, q_{T}\right)$ and $\left(A_{S}, q_{S}\right)$, respectively. Then one has an isomorphism $\mathscr{A}_{T} \rightarrow h^{\star} \mathscr{A}_{S}$ such that the following diagram commutes:

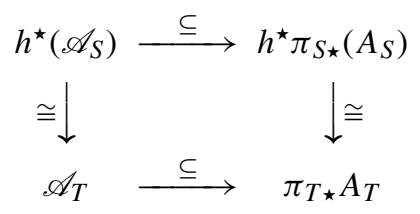

Proof. This is, in fact, a direct consequence of Diagram (2).

Semistable generalized parabolic bundles. As in the introduction, we fix a stability parameter $\alpha \in(0,1) \cap \mathbb{Q}$. A generalized parabolic bundle $(A, q)$ on the curve $\widetilde{X}$ is called $\alpha$-(semi)stable if, for every subbundle $0 \subsetneq B \subsetneq A$, the inequality

$$
\frac{\alpha-\operatorname{pardeg}(B)}{\operatorname{rk}(B)}(\leq) \frac{\alpha-\operatorname{pardeg}(A)}{\operatorname{rk}(A)}
$$

is satisfied. 
Example 2.3.2. The (semi)stability condition makes sense also for the value $\alpha=1$. In fact, the following is known:

Proposition. A generalized parabolic bundle $(A, q)$ is 1-(semi)stable if and only if the associated torsion free sheaf $\mathscr{A}$ on $X$ is (semi)stable.

Now, fix $d \in \mathbb{Z}, r \in \mathbb{Z}_{>0}$. By standard considerations, one infers

Corollary. Suppose that the stability parameter $\alpha$ is sufficiently close to one. Then, for any generalized parabolic bundle $(A, q)$ with $\operatorname{deg}(A)=d$ and $\operatorname{rk}(A)=r$ with associated torsion free sheaf $\mathscr{A}$, one has:

(i) If $(A, q)$ is $\alpha$-semistable, then $\mathscr{A}$ is semistable.

(ii) If $\mathscr{A}$ is stable, then $(A, q)$ is $\alpha$-stable.

The above proposition is Proposition 4.2 in [2]. We will come back to this in Section 5.2 within our context.

For $d$ and $r$ as above, we want to study the moduli functors

$$
\begin{aligned}
& \underline{\mathrm{GPB}}_{d / r}^{\alpha-(\mathrm{s}) \mathrm{s}}: \underline{\mathrm{Sch}}_{\mathbb{C}} \rightarrow \underline{\mathrm{Set}}, \\
& S \mapsto\left\{\begin{array}{l}
\text { equivalence classes of families } \\
\text { of } \alpha \text {-(semi)stable generalized } \\
\text { parabolic bundles of degree } d \\
\text { and rank } r \text { parameterized by } S
\end{array}\right\} .
\end{aligned}
$$

In Section 4 of [2], we then find

Theorem 2.3.3. There exist a projective scheme $\mathscr{G} \mathscr{P} \mathscr{B}_{d / r}^{\alpha \text {-ss }}$ and an open subscheme $\mathscr{G} \mathscr{P} \mathscr{B}_{d / r}^{\alpha-\mathrm{s}} \subset \mathscr{G} \mathscr{P} \mathscr{B}_{d / r}^{\alpha \text {-ss }}$ as well as natural transformations of functors

$$
\vartheta^{\alpha-(\mathrm{s}) \mathrm{s}}: \underline{\mathrm{GPB}}_{d / r}^{\alpha-(\mathrm{s}) \mathrm{s}} \rightarrow h_{\mathscr{G} \mathscr{P} \mathscr{B}_{d / r}^{\alpha-(\mathrm{s}) \mathrm{s}}}
$$

with the following properties:

1. For every scheme $\mathscr{N}$ and every natural transformation $\vartheta^{\prime}: \mathscr{G} \mathscr{P}_{d / r}^{\alpha \text { ss }} \rightarrow h_{\mathscr{N}}$, there is a unique morphism $\psi: \mathscr{G} \mathscr{P} \mathscr{B}_{d / r}^{\alpha \text {-ss }} \rightarrow \mathscr{N}$ with $\vartheta^{\prime}=h(\psi) \circ \vartheta^{\alpha \text {-ss }}$.

2. The scheme $\mathscr{G} \mathscr{P} \mathscr{B}_{d / r}^{\alpha-\mathrm{s}}$ is a coarse moduli space for the functor $\underline{\mathrm{GPB}}_{d / r}^{\alpha-\mathrm{s}}$.

For $\alpha$ sufficiently close to one, the map which associates to the equivalence class of a family $\left(A_{S}, q_{S}\right)$ of generalized parabolic bundles parameterized by $S$ the equivalence class of the associated family $\mathscr{A}_{S}$ of torsion free sheaves on $X$ defines a natural transformation of the functor $\mathrm{GPB}_{d / r}^{\alpha \text {-ss }}$ to the moduli functor of semistable torsion free sheaves on $X$, so that the theorem implies

Corollary 2.3.4. For $\alpha$ close enough to one, there is a surjective morphism

$$
\mathscr{G} \mathscr{P}_{d / r}^{\alpha-s s} \rightarrow \mathscr{U}(r, d) .
$$

Bhosle shows that this morphism is a desingularization if $(d, r)=1([2$, Theorem 3 , Section 4]). 


\section{Decorated generalized parabolic bundles}

The general strategy to construct our moduli spaces is the one adopted in our papers [16] and [17]. This means that we will first develop a theory of decorated generalized parabolic bundles in order to obtain our moduli spaces.

\subsection{The homogeneous case}

We fix non-negative integers $a, b, c$, and a line bundle $L$ on $\widetilde{X}$. A generalized parabolic bundle with a decoration of type $(a, b, c ; L)$ is a triple $(A, q, \varphi)$, where $(A, q)$ is a generalized parabolic bundle as before, and $\varphi:\left(A^{\otimes a}\right)^{\oplus b} \rightarrow \operatorname{det}(A)^{\otimes c} \otimes L$ is a non-trivial homomorphism. Two generalized parabolic bundles $\left(A_{1}, q_{1}, \varphi_{1}\right)$ and $\left(A_{2}, q_{2}, \varphi_{2}\right)$ with a decoration of type $(a, b, c ; L)$ are said to be equivalent if there are an isomorphism $\psi: A_{1} \rightarrow A_{2}$ and a number $z \in \mathbb{C}^{\star}$ such that

$$
q_{1}=q_{2} \circ\left(\psi_{\mid\left\{N_{1}, N_{2}\right\}}\right), \quad \varphi_{2}=\left(\operatorname{det}(\psi)^{\otimes c} \otimes\left(z \cdot \operatorname{id}_{L}\right)\right) \circ \varphi_{1} \circ\left(\left(\psi^{\otimes a}\right)^{\oplus b}\right)^{-1} .
$$

A family of generalized parabolic bundles with a decoration of type $(a, b, c ; L)$ parameterized by a scheme $S$ is a quadruple $\left(A_{S}, q_{S}, \mathfrak{N}_{S}, \varphi_{S}\right)$, where

- $\left(A_{S}, q_{S}\right)$ is a family of generalized parabolic bundles,

- $\mathfrak{N}_{S}$ is a line bundle on $S$,

- $\varphi_{S}:\left(A_{S}^{\otimes a}\right)^{\oplus b} \rightarrow \operatorname{det}\left(A_{S}\right)^{\otimes c} \otimes \pi_{\widetilde{X}}^{\star} L \otimes \pi_{S}^{\star} \mathfrak{N}_{S}$ is a homomorphism such that $\varphi_{S \mid\{s\} \times \widetilde{X}} \not \equiv 0$ for all $s \in S$

We will call two such families $\left(A_{S}^{1}, q_{S}^{1}, \mathfrak{N}_{S}^{1}, \varphi_{S}^{1}\right)$ and $\left(A_{S}^{2}, q_{S}^{2}, \mathfrak{N}_{S}^{2}, \varphi_{S}^{2}\right)$ equivalent if there are isomorphisms $\psi_{S}: A_{S}^{1} \rightarrow A_{S}^{2}$ and $\chi_{S}: \mathfrak{N}_{S}^{1} \rightarrow \mathfrak{N}_{S}^{2}$ such that

$$
\begin{aligned}
& q_{S}^{1}=q_{S}^{2} \circ\left(\bar{\pi}_{S_{\star}}\left(\psi_{S \mid S_{N_{1}, N_{2}}}\right)\right), \\
& \varphi_{S}^{2}=\left(\operatorname{det}\left(\psi_{S}\right)^{\otimes c} \otimes \pi_{S}^{\star}\left(\chi_{S}\right) \otimes \operatorname{id}_{\pi_{\tilde{X}}^{\star} L}\right) \circ \varphi_{S}^{1} \circ\left(\left(\psi_{S}^{\otimes a}\right)^{\oplus b}\right)^{-1} .
\end{aligned}
$$

Let $(A, q, \varphi)$ be a generalized parabolic bundle with a decoration of type $(a, b, c ; L)$. Suppose we are given a weighted filtration $\left(A^{\bullet}, \underline{\alpha}\right)$ of $A$. We then define

$$
\begin{aligned}
\mu_{a, b, c}\left(A^{\bullet}, \underline{\alpha} ; \varphi\right):=-\min \left\{\gamma_{j_{1}}+\cdots+\gamma_{j_{a}} \mid\left(j_{1}, \ldots, j_{a}\right) \in\right. & \{1, \ldots, s+1\}^{\times a}, \\
& \varphi_{\mid\left(A_{j_{1}} \otimes \cdots \otimes A_{j_{a}}\right)}{ }^{\circ b} \not \equiv 0 .
\end{aligned}
$$

In this definition, we used the weight vector

$$
\underline{\gamma}=\left(\gamma_{1}, \ldots, \gamma_{s+1}\right):=\sum_{i=1}^{s} \alpha_{i} \cdot(\underbrace{\operatorname{rk}\left(A_{i}\right)-r, \ldots, \operatorname{rk}\left(A_{i}\right)-r}_{\operatorname{rk}\left(A_{i}\right) \times}, \underbrace{\operatorname{rk}\left(A_{i}\right), \ldots, \operatorname{rk}\left(A_{i}\right)}_{\left(r-\operatorname{rk}\left(A_{i}\right)\right) \times}),
$$

where $r:=\operatorname{rk}(A)$.

Remark 3.1.1. This definition agrees with the one given by the author in [15, Lemma $1.4]$, as one easily checks. 
We fix numbers $\alpha \in(0,1) \cap \mathbb{Q}$ and $\delta \in \mathbb{Q}_{>0}$. Then the decorated generalized parabolic bundle $(A, q, \varphi)$ is said to be $(\alpha, \delta)$-(semi)stable if for every weighted filtration $\left(A^{\bullet}, \underline{\alpha}\right)$ of $A$, the inequality

$$
P_{\alpha}\left(A^{\bullet}, \underline{\alpha}\right)+\delta \cdot \mu_{a, b, c}\left(A^{\bullet}, \underline{\alpha} ; \varphi\right)(\geq) 0
$$

is satisfied. Now, we may introduce the moduli functors

$$
\begin{aligned}
\underline{\mathrm{DGPB}}_{d / r / a / b / c / L}^{(\alpha, \delta)-(\mathrm{s}) \mathrm{s}}: \underline{\mathrm{Sch}}_{\mathbb{C}} \rightarrow \frac{\text { Set }}{} \\
S \mapsto\left\{\begin{array}{l}
\text { equivalence classes of families } \\
\text { of }(\alpha, \delta) \text {-(semi)stable } \\
\text { generalized parabolic bundles } \\
\left(A_{S}, q_{S}, \mathfrak{N}_{S}, \varphi_{S}\right) \text { with a } \\
\text { decoration of type }(a, b, c ; L) \\
\text { such that } \operatorname{deg}\left(A_{S \mid\{s\} \times X}\right)=d, \\
\text { for all } s \in S, \text { and } \operatorname{rk}\left(A_{S}\right)=r
\end{array}\right\} .
\end{aligned}
$$

Our main auxiliary result is

Theorem 3.1.2. There exist a projective scheme $\mathscr{D} \mathscr{G} \mathscr{P} \mathscr{B}_{d / r / a / b / c / L}^{(\alpha, \delta)-(\mathrm{s}) \mathrm{s}}$ and an open subscheme $\mathscr{D} \mathscr{G} \mathscr{P} \mathscr{B}_{d / r / a / b / c / L}^{(\alpha, \delta)-s} \subset \mathscr{D} \mathscr{G} \mathscr{P}_{d / r / a / b / c / L}^{(\alpha, \delta) \text { ss }}$ as well as natural transformations of functors

$$
\vartheta^{(\alpha, \delta)-(\mathrm{s}) \mathrm{s}}: \underline{\operatorname{DGPB}}_{d / r / a / b / c / L}^{(\alpha, \delta)-(\mathrm{s}) \mathrm{s}} \rightarrow h_{\mathscr{D} \mathscr{G} \mathscr{P}_{d / r / a / b / c / L}^{(\alpha, \delta)-(\mathrm{s}) \mathrm{s}}}
$$

with the following properties:

(i) For every $\mathscr{N}$ and every natural transformation $\vartheta^{\prime}: \underline{\mathrm{DGPB}}_{d / r / a / b / c / L}^{(\alpha, \delta)-\mathrm{ss}} \rightarrow h_{\mathscr{N}}$, there is a unique morphism $\psi: \mathscr{D} \mathscr{G} \mathscr{P}_{d / r / a / b / c / L}^{(\alpha, \delta) \text { ss }} \rightarrow \mathscr{N}$ with $\vartheta^{\prime}=h(\psi) \circ \vartheta^{(\alpha, \delta) \text {-ss }}$.

(ii) The scheme $\mathscr{D} \mathscr{G} \mathscr{P} \mathscr{B}_{d / r / a / b / c / L}^{(\alpha, \delta)-s}$ is a coarse moduli space for the functor $\underline{\operatorname{DGPB}}_{d / r / a / b / c / L}^{(\alpha, \delta)-\mathrm{s}}$.

\subsection{The inhomogeneous case}

Here, we will make some remarks concerning a different class of decorations which we will need in some places, but we do not have to develop the whole moduli theory. This time, we fix tuples $\underline{a}=\left(a_{1}, \ldots, a_{n}\right), \underline{b}=\left(b_{1}, \ldots, b_{n}\right)$, and $\underline{c}=\left(c_{1}, \ldots, c_{n}\right)$ of nonnegative integers such that $a_{i}-r c_{i}>0$ for $i=1, \ldots, n$. If $a_{i}-r c_{i}=a_{j}-r c_{j}, 1 \leq i<$ $j \leq n$, we call the triple $(\underline{a}, \underline{b}, \underline{c})$ homogeneous. The objects we will study now are triples $(A, q, \varphi)$ with $(A, q)$ a generalized parabolic bundle of rank $r$ and $\varphi: A_{\underline{a}, \underline{b}, \underline{c}} \rightarrow \mathscr{O}_{\widetilde{X}}$ a non-trivial homomorphism. Here,

$$
A_{\underline{a}, \underline{b}, \underline{c}}:=\bigoplus_{i=1}^{n}\left(A^{\otimes a_{i}}\right)^{\oplus b_{i}} \otimes\left(\operatorname{det}(A)^{\vee}\right)^{\otimes c_{i}} .
$$


We call $(A, q, \varphi)$ a generalized parabolic bundle with a decoration of type $(\underline{a}, \underline{b}, \underline{c})$, and we say that $\left(A_{1}, q_{1}, \varphi_{1}\right)$ is equivalent to $\left(A_{2}, q_{2}, \varphi_{2}\right)$ if there is an isomorphism $\psi: A_{1} \rightarrow$ $A_{2}$ such that

$$
q_{1}=q_{2} \circ\left(\psi_{\mid\left\{N_{1}, N_{2}\right\}}\right), \quad \varphi_{1}=\varphi_{2} \circ \psi_{\underline{a}, \underline{b}, \underline{c}},
$$

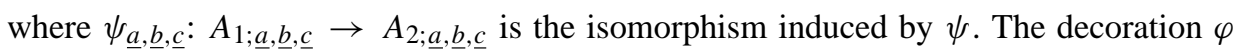
breaks into components

$$
\varphi_{i}:\left(A^{\otimes a_{i}}\right)^{\oplus b_{i}} \rightarrow \operatorname{det}(A)^{\otimes c_{i}}, \quad i=1, \ldots, n .
$$

Given a weighted filtration $\left(A^{\bullet}, \underline{\alpha}\right)$, we set, for $A_{s+1}:=A$ and $i=1, \ldots, n$,

$$
\begin{aligned}
\mu\left(A^{\bullet}, \underline{\alpha} ; \varphi_{i}\right):=-\min \left\{\gamma_{j_{1}}+\cdots+\gamma_{j_{a_{i}}} \mid\left(j_{1}, \ldots, j_{a_{i}}\right) \in\{1, \ldots, s+1\}^{\times a_{i}},\right. & \left.\varphi_{i \mid\left(A_{j_{1}} \otimes \cdots \otimes A_{j a_{i}}\right.}{ }^{\oplus b_{i}} \not \equiv 0\right\}
\end{aligned}
$$

as well as

$$
\mu_{\underline{a}, \underline{b}, \underline{c}}\left(A^{\bullet}, \underline{\alpha} ; \varphi\right):=\max \left\{\mu\left(A^{\bullet}, \underline{\alpha} ; \varphi_{i}\right) \mid i=1, \ldots, n\right\} .
$$

Next, we will relate inhomogeneous decorations to homogeneous ones. Let $v_{1}<$ $\cdots<v_{m}$ be the integers which occur as $a_{i}-r c_{i}, i=1, \ldots, n$. Given any three non-negative integers $a, b$, and $c$, we write $V_{a, b, c}$ for the GL $(V)$-module $\left(V^{\otimes a}\right)^{\oplus b} \otimes$ $\left(\bigwedge^{\operatorname{dim}(V)} V\right)^{\otimes-c}$. Set $V_{j}:=\bigoplus_{i: a_{i}-r c_{i}=v_{j}} V_{a_{i}, b_{i}, c_{i}}, j=1, \ldots, m$. Choose a sufficiently large common multiple $\omega$ of $v_{1}, \ldots, v_{m}$. If we let $\mathbb{C}^{\star}$ act on $V_{j}$ by multiplication with $z^{v_{j}}$, the weighted projective space $\left(V_{\underline{a}, \underline{b}, \underline{c}} \backslash\{0\}\right) / \mathbb{C}^{\star}$ gets embedded into $\mathbb{P}\left(\mathbb{V}_{\omega}\right)$,

$$
\mathbb{V}_{\omega}:=\bigoplus_{\substack{\left(d_{1}, \ldots, d_{m}\right): d_{i} \geq 0, v_{1} d_{1}+\cdots+v_{m} d_{m}=\omega}} S^{d_{1}}\left(V_{1}^{\vee}\right) \otimes \cdots \otimes S^{d_{m}}\left(V_{m}^{\vee}\right)
$$

The details may be found in [17]. We may find positive integers $D, E, F$ with $D-r F=$ $-\omega$ such that $\mathbb{V}_{\omega}$ is a direct summand of $V_{D, E, F}([15$, Corollary 1.2]) and we have an embedding $\mathbb{P}\left(\mathbb{V}_{\omega}\right) \hookrightarrow \mathbb{P}\left(V_{D, E, F}\right)$.

Let $(A, q, \varphi)$ be a generalized parabolic bundle with a decoration of type $(\underline{a}, \underline{b}, \underline{c})$. Then $\varphi$ defines, for every tuple $\underline{d}=\left(d_{1}, \ldots, d_{m}\right)$ with $v_{1} d_{1}+\cdots+v_{m} d_{m}=\omega$, a homomorphism

$$
\tilde{\varphi}_{\underline{d}}: S^{d_{1}}\left(B_{1}\right) \otimes \cdots \otimes S^{d_{m}}\left(B_{m}\right) \rightarrow \mathscr{O}_{X}
$$

where $B_{j}:=\bigoplus_{i: a_{i}-r c_{i}=v_{j}} A_{a_{i}, b_{i}, c_{i}}, j=1, \ldots, m$. These homomorphisms add up to

$$
\tilde{\varphi}: \bigoplus_{\substack{\left(d_{1}, \ldots, d_{m}\right): d_{i} \geq 0, v_{1} d_{1}+\cdots+v_{m} d_{m}=\omega}} S^{d_{1}}\left(B_{1}\right) \otimes \cdots \otimes S^{d_{m}}\left(B_{m}\right) \rightarrow \mathscr{O}_{\tilde{X}} .
$$

This finally defines

$$
\widehat{\varphi}:\left(A^{\otimes D}\right)^{\oplus E} \rightarrow \operatorname{det}(A)^{\otimes F} .
$$

For every weighted filtration $\left(A^{\bullet}, \underline{\alpha}\right)$ of $A$, we set

$$
v_{\underline{a}, \underline{b}, \underline{c}}\left(A^{\bullet}, \underline{\alpha} ; \varphi\right):=\frac{1}{\omega} \cdot \mu_{D, E, F}\left(A^{\bullet}, \underline{\alpha} ; \widehat{\varphi}\right) .
$$


Again, we fix parameters $\alpha \in(0,1) \cap \mathbb{Q}$ and $\delta \in \mathbb{Q}_{>0}$. Then a generalized parabolic bundle $(A, q, \varphi)$ with a decoration of type $(\underline{a}, \underline{b}, \underline{c})$ is called $(\alpha, \delta)$-(semi)stable if, for every weighted filtration $\left(A^{\bullet}, \underline{\alpha}\right)$ of $A$, the inequality

$$
P_{\alpha}\left(A^{\bullet}, \underline{\alpha}\right)+\delta \cdot v_{\underline{a}, \underline{b}, \underline{c}}\left(A^{\bullet}, \underline{\alpha} ; \varphi\right)(\geq) 0
$$

is satisfied.

Remark 3.2.1. An obvious but important fact is

$$
v_{\underline{a}, \underline{b}, \underline{c}}\left(A^{\bullet}, \underline{\alpha} ; \varphi\right)<(=/>) 0 \Leftrightarrow \mu_{\underline{a}, \underline{b}, \underline{c}}\left(A^{\bullet}, \underline{\alpha} ; \varphi\right)<(=/>) 0 .
$$

3.3. The semistability concept for decorated generalized parabolic bundles for large values of the semistability parameter

In this section, we will explain how the concept of $(\alpha, \delta)$-(semi)stability simplifies and stabilizes when the parameter $\delta$ gets very large. We may confine ourselves to a sketch of the arguments, because they are a trivial adaptation of those in our paper [18]. As usual, we fix the input data $a, b, c, d, r$, and $L$.

Let $A$ be a locally free sheaf of degree $d$ and rank $r$ on the smooth curve $\tilde{X}$, and $\varphi:\left(A^{\otimes a}\right)^{\oplus b} \rightarrow \operatorname{det}(A)^{\otimes c} \otimes L$ a non-trivial homomorphism. Given a trivialization $\psi_{\eta}:$ $A \otimes_{\mathscr{O}_{\widetilde{X}}} K \cong \mathbb{C}^{r} \otimes_{\mathbb{C}} K$, the homomorphism $\varphi_{\mid \eta}$ defines an element $\widetilde{\sigma}_{\eta} \in \mathbb{P}\left(V_{a, b, c}\right) \times_{\operatorname{Spec}(\mathbb{C})}$ $\operatorname{Spec}(K)$. Here, $\eta$ is the generic point of $\widetilde{X}$ and $K$ is the function field of $\widetilde{X}$. We say that $\varphi$ is generically semistable if $\widetilde{\sigma}_{\eta} \in \mathbb{P}_{a, b, c}^{\mathrm{ss}} \times \operatorname{Spec}(\mathbb{C}) \operatorname{Spec}(K)$, with $\mathbb{P}_{a, b, c}^{\mathrm{ss}}$ the set of SL(V)semistable points in $\mathbb{P}\left(V_{a, b, c}\right)$.

Remark 3.3.1. (i) The above notion of generic semistability does not depend on the choice of the trivialization. In fact, set $\mathbb{A}:=A_{\mid \eta}$. This is an $r$-dimensional $K$-vector space. The condition is that $\left[\varphi_{\eta}\right] \in \mathbb{P}\left(\mathbb{A}_{a, b, c}\right)$ be contained in the set of points which are semistable for the action of the group $\operatorname{SL}(\mathbb{A})$. This follows from Proposition 1.14 in Chapter 1.4 of [12].

(ii) With the above formulation of generic semistability and the definition of $\mu_{a, b, c}\left(A^{\bullet}, \underline{\alpha} ; \varphi\right)$ in the form of Lemma 1.4 of [15], it is obvious that

$$
\mu_{a, b, c}\left(A^{\bullet}, \underline{\alpha} ; \varphi\right) \geq 0
$$

for every weighted filtration $\left(A^{\bullet}, \underline{\alpha}\right)$ of $A$, if $\varphi$ is generically semistable.

Now, fix a rational number $\alpha \in(0,1)$. We say that a generalized parabolic bundle $(A, q, \varphi)$ with a decoration of type $(a, b, c ; L)$ is $\alpha$-asymptotically (semi)stable if (a) $\varphi$ is generically semistable and (b) for every weighted filtration $\left(A^{\bullet}, \underline{\alpha}\right)$ of $A$ such that $\mu_{a, b, c}\left(A^{\bullet}, \underline{\alpha} ; \varphi\right)=0$, one has

$$
P_{\alpha}\left(A^{\bullet}, \underline{\alpha}\right)(\geq) 0
$$


Theorem 3.3.2. Given $a, b, c, d, r, L$ and $\alpha$ as above, there is a positive rational number $\delta_{\infty}$ such that for every generalized parabolic bundle $(A, q, \varphi)$ with a decoration of type $(a, b, c ; L)$, satisfying $\operatorname{deg}(E)=d$ and $\operatorname{rk}(E)=r$, and every rational number $\delta>\delta_{\infty}$, the following two conditions are equivalent:

(i) $(A, q, \varphi)$ is $(\alpha, \delta)-($ semi $)$ stable.

(ii) $(A, q, \varphi)$ is $\alpha$-asymptotically (semi)stable.

Proof. The proof is subdivided into several steps as in [18].

Step 1. Here, one proves the following result.

Proposition 3.3.3. There is a positive rational number $\delta_{0}$ such that, for every $\delta>\delta_{0}$, a generalized parabolic bundle $(A, q, \varphi)$ with a decoration of type $(a, b, c ; L), \operatorname{deg}(A)=d$, and $\operatorname{rk}(A)=r$ which is $(\alpha, \delta)$-semistable is $\alpha$-asymptotically semistable as well.

Proof. Note that it suffices to show that, for large $\delta, \varphi$ is generically semistable, because then $(\alpha, \delta)$-semistability obviously implies $\alpha$-asymptotic semistability, by Remark 3.3.1(ii). If $\varphi$ is not generically semistable, then the proof of Theorem 3.1.1 in [18] shows that there are a constant $C$ (which depends only on the input data) and a weighted filtration $\left(A^{\bullet}, \underline{\alpha}\right)$ with

$$
C \geq \sum_{i=1}^{s} \alpha_{i}\left(\operatorname{rk} A_{i} \cdot \operatorname{deg}(A)-r \cdot \operatorname{deg}\left(A_{i}\right)\right)
$$

and

$$
\mu_{a, b, c}\left(A^{\bullet}, \underline{\alpha} ; \varphi\right)<0 \text {. }
$$

Furthermore, the tuple ( $\operatorname{rk} A_{1}, \ldots, \mathrm{rk} A_{s} ; \alpha_{1}, \ldots, \alpha_{s}$ ) belongs to a finite set $\mathscr{T}$ which depends only on $a, b$, and $c$ ([18, Remark 2.1.4(ii)]). Since, by definition of the $\alpha$-parabolic degree,

$$
\begin{aligned}
\operatorname{rk} A^{\prime} \cdot & \alpha-\operatorname{pardeg}(A)-r \cdot \alpha-\operatorname{pardeg}\left(A^{\prime}\right) \\
& =\operatorname{rk} A^{\prime} \cdot \operatorname{deg}(A)-r \cdot \operatorname{deg}\left(A^{\prime}\right)-\alpha \cdot r \cdot \operatorname{rk} A^{\prime}+\alpha \cdot r \cdot \operatorname{dim} q\left(A_{N_{1}}^{\prime} \oplus A_{N_{2}}^{\prime}\right) \\
& \leq \operatorname{rk} A^{\prime} \cdot \operatorname{deg}(A)-r \cdot \operatorname{deg}\left(A^{\prime}\right)+\alpha \cdot r \cdot(r-1)
\end{aligned}
$$

for any subbundle $A^{\prime}$ of $A$, it is clear that we can find a constant $C^{\prime}$ which depends only on the input data with

$$
C^{\prime} \geq \sum_{i=1}^{s} \alpha_{i}\left(\operatorname{rk} A_{i} \cdot \alpha-\operatorname{pardeg}(A)-r \cdot \alpha-\operatorname{pardeg}\left(A_{i}\right)\right)
$$

Applying the condition of $(\alpha, \delta)$-semistability to the above weighted filtration, we find the estimate

$$
C^{\prime}-\delta \geq P_{\alpha}\left(A^{\bullet}, \underline{\alpha}\right)+\delta \cdot \mu_{a, b, c}\left(A^{\bullet}, \underline{\alpha} ; \varphi\right) \geq 0,
$$

so that the proposition holds indeed for $\delta_{0}:=C^{\prime}$. 
Step 2

Proposition 3.3.4. The set of isomorphy classes [A] of vector bundles A of rank $r$ and degree $d$ for which there exists a non-trivial homomorphism $\varphi:\left(A^{\otimes a}\right)^{\oplus b} \rightarrow \operatorname{det}(A)^{\otimes c} \otimes L$ such that $(A, \varphi)$ is $\alpha$-asymptotically semistable, is bounded.

Proof. First, we note that the arguments given in the section entitled "Simplification of the semistability concept for decorated sheaves" in [18] apply without modification to our situation, too. This means that there is a finite set

$$
\mathscr{T}=\left\{\left(r_{1}^{i}, \ldots, r_{s(i)}^{i} ; \alpha_{1}^{i}, \ldots, \alpha_{s(i)}^{i}\right) \mid i=1, \ldots, t\right\}
$$

such that the following holds true.

Proposition 3.3.5. Given any positive rational number $\delta$, a generalized parabolic bundle $(A, q, \varphi)$ with a decoration of type $(a, b, c ; L)$ is $(\alpha, \delta)$-(semi)stable if and only if the condition

$$
P_{\alpha}\left(A^{\bullet}, \underline{\alpha}\right)+\delta \cdot \mu_{a, b, c}\left(A^{\bullet}, \underline{\alpha} ; \varphi\right)(\geq) 0
$$

holds for every weighted filtration $\left(A^{\bullet}, \underline{\alpha}\right)$ of A satisfying

$$
\left(\operatorname{rk} A_{1}, \ldots, \operatorname{rk} A_{s} ; \alpha_{1}, \ldots, \alpha_{s}\right) \in \mathscr{T} \text {. }
$$

With this definition at hand, the proof of Proposition 3.3.4 may be adapted from the proof of Proposition 3.2.2 in [18] in very much the same fashion as the proof of Proposition 3.3.3 has been adapted from the proof of Theorem 3.1.1 in [18].

Step 3. Assume $\delta>\delta_{0}$. Then one direction is taken care of by Proposition 3.3.3 but we still have to show that an $\alpha$-asymptotically (semi)stable decorated generalized parabolic bundle $(A, q, \varphi)$ satisfies the condition of $(\alpha, \delta)$-(semi)stability for all $\delta \gg 0$. Recall that $\mu_{a, b, c}\left(A^{\bullet}, \underline{\alpha} ; \varphi\right) \geq 0$ for any weighted filtration $\left(A^{\bullet}, \underline{\alpha}\right)$ (Remark 3.3.1 (ii)). Moreover, the condition of $(\alpha, \delta)$-(semi)stability is satisfied for those weighted filtrations $\left(A^{\bullet}, \underline{\alpha}\right)$ with $\mu_{a, b, c}\left(A^{\bullet}, \underline{\alpha} ; \varphi\right)=0$. Finally, we have to check what happens for weighted filtrations $\left(A^{\bullet}, \underline{\alpha}\right)$ with $\mu_{a, b, c}\left(A^{\bullet}, \underline{\alpha} ; \varphi\right)>0$. By Proposition 3.3.1, we may assume

$$
\left(\operatorname{rk} A_{1}, \ldots, \operatorname{rk} A_{s} ; \alpha_{1}, \ldots, \alpha_{s}\right) \in \mathscr{T} \text {. }
$$

The boundedness result 3.3.4 implies that we may find a constant $C^{\prime \prime}$ with

$$
P_{\alpha}\left(A^{\bullet}, \underline{\alpha}\right) \geq C^{\prime \prime}
$$

for any weighted filtration $A^{\bullet}, \underline{\alpha}$ of $A$ for which $(4)$ holds. Suppose $\delta>-C^{\prime \prime}$. Then

$$
P_{\alpha}\left(A^{\bullet}, \underline{\alpha}\right)+\delta \cdot \mu_{a, b, c}\left(A^{\bullet}, \underline{\alpha} ; \varphi\right) \geq C^{\prime \prime}+\delta>0,
$$

and we are done. 


\section{Descending principal $G$-bundles}

Let $G$ be a semisimple linear algebraic group over the complex numbers, and fix a faithful representation $\varrho: G \rightarrow \mathrm{GL}(V)$ on the $r$-dimensional complex vector space $V$.

\subsection{The varieties $\operatorname{Hom}\left(\mathbb{C}^{r}, V^{\vee}\right) / / G$ and $\mathbb{P}\left(\operatorname{Hom}\left(\mathbb{C}^{r}, V^{\vee}\right)^{\vee}\right) / / G$}

We obtain the representation

$$
\begin{aligned}
R: \mathrm{GL}_{r}(\mathbb{C}) \times G & \rightarrow \mathrm{GL}\left(\mathbb{C}^{r} \otimes V\right), \\
\left(g, g^{\prime}\right) & \mapsto\left(w \otimes v \mapsto(g \cdot w) \otimes \varrho\left(g^{\prime}\right)(v)\right),
\end{aligned}
$$

as well as the actions induced by the contragredient representation $R^{\vee}$,

$$
\Gamma:\left(\mathrm{GL}_{r}(\mathbb{C}) \times G\right) \times \operatorname{Hom}\left(\mathbb{C}^{r}, V^{\vee}\right) \rightarrow \operatorname{Hom}\left(\mathbb{C}^{r}, V^{\vee}\right)
$$

and

$$
\bar{\Gamma}:\left(\mathrm{GL}_{r}(\mathbb{C}) \times G\right) \times \mathbb{P}\left(\operatorname{Hom}\left(\mathbb{C}^{r}, V^{\vee}\right)^{\vee}\right) \rightarrow \mathbb{P}\left(\operatorname{Hom}\left(\mathbb{C}^{r}, V^{\vee}\right)^{\vee}\right) .
$$

For any degree $d \geq 0$, the representation $R$ induces an action of $\mathrm{GL}_{r}(\mathbb{C}) \times G$ on the symmetric power $\operatorname{Sym}^{d}\left(\mathbb{C}^{r} \otimes V\right)$, so that there is an action of $\mathrm{GL}_{r}(\mathbb{C}) \times G$ on the algebra $\operatorname{Sym}^{\star}\left(\mathbb{C}^{r} \otimes V\right)$. We also get the induced action of $\mathrm{GL}_{r}(\mathbb{C})$ on the algebra $\operatorname{Sym}^{\star}\left(\mathbb{C}^{r} \otimes V\right)^{G}$ of $G$-invariants in $\operatorname{Sym}^{\star}\left(\mathbb{C}^{r} \otimes V\right)$. This $\mathrm{GL}_{r}(\mathbb{C})$-action on $\operatorname{Sym}^{\star}\left(\mathbb{C}^{r} \otimes V\right)^{G}$ is, by construction, the same which is induced by the $\mathrm{GL}_{r}(\mathbb{C})$-action $\widetilde{\Gamma}: G \times \mathbb{H} \rightarrow \mathbb{H}$, $\mathbb{H}:=\operatorname{Hom}\left(\mathbb{C}^{r}, V^{\vee}\right) / / G$, coming from $\Gamma$. Furthermore, we note that, for any $d \geq 0$, $\operatorname{Sym}^{d}\left(\mathbb{C}^{r} \otimes V\right)^{G}$ is a GL $(\mathbb{C})$-submodule of $\operatorname{Sym}^{d}\left(\mathbb{C}^{r} \otimes V\right)$ and, thus, also of $\left(\mathbb{C}^{r} \otimes V\right)^{\otimes d}$, so that we find $\mathrm{GL}_{r}(\mathbb{C})$-module homomorphisms

$$
\pi_{d}:\left(\mathbb{C}^{r} \otimes V\right)^{\otimes d} \rightarrow \operatorname{Sym}^{d}\left(\mathbb{C}^{r} \otimes V\right)^{G}, \quad d \geq 0 .
$$

Choose an $s$ so large that $\bigoplus_{i=0}^{s} \operatorname{Sym}^{i}\left(\mathbb{C}^{r} \otimes V\right)^{G}$ contains a set of generators for the algebra $\operatorname{Sym}^{\star}\left(\mathbb{C}^{r} \otimes V\right)^{G}$. We define the $\mathrm{GL}_{r}(\mathbb{C})$-module

$$
\mathbb{W}_{s}:=\bigoplus_{i=1}^{s} \mathbb{U}_{i}, \quad \mathbb{U}_{d}:=\left(\left(\mathbb{C}^{r} \otimes V\right)^{\otimes d}\right)^{\vee}, \quad d \geq 0 .
$$

The homomorphisms $\pi_{d}, d \geq 0$, provide us with an algebra homomorphism

$$
\pi: \operatorname{Sym}^{\star}\left(\mathbb{W}_{s}^{\vee}\right) \rightarrow \operatorname{Sym}^{\star}\left(\mathbb{C}^{r} \otimes V\right)^{G}
$$

which is compatible with the $\mathrm{GL}_{r}(\mathbb{C})$-actions. To summarize, we have found a representation $\kappa_{s}: \mathrm{GL}_{r}(\mathbb{C}) \rightarrow \mathrm{GL}\left(\mathbb{W}_{s}\right)$ and a $\mathrm{GL}_{r}(\mathbb{C})$-equivariant closed embedding

$$
\iota: \mathbb{H} \hookrightarrow \mathbb{W}_{s} .
$$

We have to analyze the semistability of points $\iota(h), h \in \mathbb{H}$, with respect to the action of the special linear group $\operatorname{SL}_{r}(\mathbb{C})$. Set $\mathbb{I}:=\operatorname{Isom}\left(\mathbb{C}^{r}, V^{\vee}\right) / G$. This is a dense open subset of $\mathbb{H}$. 
Lemma 4.1.1. (i) Every point $\iota(i), i \in \mathbb{I}$, is $\mathrm{SL}_{r}(\mathbb{C})$-polystable.

(ii) No point $\iota(h), h \in \mathbb{H} \backslash \mathbb{I}$, is $\mathrm{SL}_{r}(\mathbb{C})$-semistable.

Proof. This is Lemma 4.1.1 in [17]. We briefly recall the argument.

(i) We choose a basis for $V^{\vee}$. This provides us with the $\left(\mathrm{SL}_{r}(\mathbb{C}) \times G\right)$-invariant function $\mathfrak{d}: \operatorname{Hom}\left(\mathbb{C}^{r}, V^{\vee}\right) \rightarrow \mathbb{C}, f \mapsto \operatorname{det}(f)$, which descends to a (non-constant) function on $\mathbb{H}$, called again $\mathfrak{d}$. For any $i \in \mathbb{I}$, we clearly have $\mathfrak{d}(\iota(i)) \neq 0$, so that $\iota(i)$ is $\mathrm{SL}_{r}(\mathbb{C})$ semistable. Furthermore, for any $f \in \operatorname{Isom}\left(\mathbb{C}^{r}, V^{\vee}\right)$, the $\left(\mathrm{SL}_{r}(\mathbb{C}) \times G\right)$-orbit of $f$ is just the level set $\mathfrak{d}^{-1}(z)$ for an appropriate $z \in \mathbb{C}^{\star}$. In particular, it is closed. The image of this orbit is the $\mathrm{SL}_{r}(\mathbb{C})$-orbit of $i:=[f]$ in $\mathbb{H}$, which is, therefore, closed. Since $\iota$ is a closed, $\mathrm{SL}_{r}(\mathbb{C})$-equivariant embedding, the orbit of $\iota(i)$ is also closed.

(ii) It is obvious from the construction that the ring of $\mathrm{SL}_{r}(\mathbb{C})$-invariant functions on $\mathbb{H}$ is generated by $\mathfrak{d}$. This makes the asserted property evident.

Next, we study the quotient

$$
\overline{\mathbb{H}}:=\mathbb{P}\left(\operatorname{Hom}\left(\mathbb{C}^{r}, V^{\vee}\right)^{\vee}\right) / / G=(\mathbb{H} \backslash\{0\}) / / \mathbb{C}^{\star} .
$$

We form the $\mathrm{GL}_{r}(\mathbb{C})$-module

$$
\mathbb{V}_{s}:=\bigoplus_{\substack{\left(d_{1}, \ldots, d_{s}\right): \\ d_{i} \geq 0, \sum i d_{i}=s !}} \operatorname{Sym}^{d_{1}}\left(\mathbb{U}_{1}^{\vee}\right) \otimes \cdots \otimes \operatorname{Sym}^{d_{s}}\left(\mathbb{U}_{s}^{\vee}\right)
$$

Then we have the $\mathrm{GL}_{r}(\mathbb{C})$-equivariant embedding

$$
\bar{\imath}:(\mathbb{H} \backslash\{0\}) / / \mathbb{C}^{\star} \hookrightarrow\left(\mathbb{W}_{S} \backslash\{0\}\right) / / \mathbb{C}^{\star} \hookrightarrow \mathbb{P}\left(\mathbb{V}_{s}\right)
$$

Note that, for appropriate $N \in \mathbb{Z}_{>0}$, we have a surjection

$$
\left(\left(\mathbb{C}^{r} \otimes V\right)^{\otimes s !}\right)^{\oplus N} \rightarrow \mathbb{V}_{s}
$$

It is again elementary that, for a point $w \in \mathbb{W}_{s} \backslash\{0\}$ and a one-parameter subgroup $\lambda: \mathbb{C}^{\star} \rightarrow \mathrm{SL}_{r}(\mathbb{C})$,

$$
\begin{aligned}
\mu_{\mathscr{O}_{\mathbb{P}\left(\left(\left(\mathbb{C}^{r} \otimes V\right) \otimes s !\right) \oplus N\right)}(1)}(\lambda,[w])=\mu_{\mathscr{O}_{\mathbb{P}\left(\mathbb{V}_{s}\right)}(1)}(\lambda,[w])< & (=/>) 0 \\
& \Leftrightarrow \mu(\lambda, w)<(=/>) 0 .
\end{aligned}
$$

Corollary 4.1.2. The point $\bar{\imath}([h]) \in \mathbb{P}\left(\mathbb{V}_{s}\right), h \in \mathbb{H}$, is $\mathrm{SL}_{r}(\mathbb{C})$-semistable if and only if $h \in \mathbb{I}$.

Proof. This follows from Lemma 4.1.1 and (5), given the obvious fact that $[\iota(h)]=$ $\bar{\imath}([h])$. 
4.2. The homogeneous and the inhomogeneous decoration associated with a singular G-bundle

A singular principal $G$-bundle on $\tilde{X}$ is a pair $(A, \widetilde{\tau})$ which consists of a vector bundle $A$ on $\widetilde{X}$ of rank $r$ with trivial determinant and a homomorphism $\tilde{\tau}: \operatorname{Sym}^{\star}(A \otimes V)^{G} \rightarrow \mathscr{O}_{\widetilde{X}}$ which is non-trivial in the sense that it is not just the projection onto the degree zero component. The basic features of this notion are outlined in our paper [16]. In particular, we recall the following alternative (see [16, Corollary 3.4]).

\section{Lemma 4.2.1. Let}

$$
\tilde{\sigma}: \tilde{X} \rightarrow \mathscr{H} o m\left(A, V^{\vee} \otimes \mathscr{O}_{\tilde{X}}\right) / / G=\mathscr{S p e c}\left(\operatorname{Sym}^{\star}(A \otimes V)^{G}\right)
$$

be the section defined by $\tilde{\tau}$. Then $\widetilde{\sigma}(\tilde{X})$ is either contained in $\mathscr{I}_{\text {som }}\left(A, V^{\vee} \otimes \mathscr{O}_{\tilde{X}}\right) / G$ or in the complement of this set.

In the former case, we call $(A, \widetilde{\tau})$ abusively a principal $G$-bundle, because it defines via the pullback diagram (1) a principal $G$-bundle $\mathscr{P}$ in the usual sense. We choose an $s \gg 0$ as in the previous section. Then to any singular $G$-bundle $(A, \tilde{\tau})$, we associate the pair $(A, \varphi)$ with

$$
\varphi: \bigoplus_{i=1}^{s}(A \otimes V)^{\otimes i} \rightarrow \operatorname{Sym}^{\star}(A \otimes V)^{G} \stackrel{\tilde{\tau}}{\rightarrow} \mathscr{O}_{\tilde{X}}
$$

and the pair $(A, \widehat{\varphi})$ with

$$
\widehat{\varphi}:\left((A \otimes V)^{\otimes s !}\right)^{\oplus N} \rightarrow \bigoplus \operatorname{Sym}^{d_{1}}(A \otimes V) \otimes \cdots \otimes \operatorname{Sym}^{d_{s}}\left((A \otimes V)^{\otimes s}\right) \rightarrow \mathscr{O}_{\tilde{X}},
$$

where the direct sum runs over all $\left(d_{1}, \ldots, d_{s}\right)$ such that $d_{i} \geq 0, i=1, \ldots, s$, and $\sum i d_{i}=s$ !, and the second arrow is

$$
\sum_{\substack{\left(d_{1}, \ldots, d_{s}\right): \\ d_{i} \geq 0, \sum i d_{i}=s !}} \operatorname{Sym}^{d_{1}}\left(\varphi_{\mid(A \otimes V)}\right) \otimes \cdots \otimes \operatorname{Sym}^{d_{s}}\left(\varphi_{\mid(A \otimes V)} \otimes s\right) .
$$

We need the following important fact.

Proposition 4.2.2. Suppose $(A, \tilde{\tau})$ is a principal $G$-bundle. Then the following conditions on a weighted filtration $\left(A^{\bullet}, \underline{\alpha}\right)$ are equivalent:

(i) $\mu\left(A^{\bullet}, \underline{\alpha}, \widehat{\varphi}\right)=0$.

(ii) $\mu\left(A^{\bullet}, \underline{\alpha}, \varphi\right)=0$.

(iii) There exists a reduction $\beta$ of the $G$-bundle $(A, \tilde{\tau})$ to a one-parameter subgroup $\lambda$ of $G$ such that $\left(A^{\bullet}, \underline{\alpha}\right)=\left(A_{\beta}^{\bullet}, \underline{\alpha}_{\beta}\right)$.

Proof. The equivalence of (i) and (ii) is a special case of (3). For the equivalence of (ii) and (iii), we refer the reader to [17, Lemma 4.2.1 and Proposition 4.2.2]. 
Next, a singular G-bundle with a generalized parabolic structure (GPS) is a triple $(A, q, \tilde{\tau})$, where $(A, q)$ is a generalized parabolic bundle of rank $r$ and $(A, \tilde{\tau})$ is a singular $G$-bundle.

Fix the semistability parameters $\alpha \in(0,1) \cap \mathbb{Q}$ and $\delta \in \mathbb{Q}_{>0}$. Then a singular $G$ bundle $(A, q, \tilde{\tau})$ with a GPS is said to be $(\alpha, \delta)$-(semi)stable if for every weighted filtration $\left(A^{\bullet}, \underline{\alpha}\right)$ of $A$ the inequality

$$
P_{\alpha}\left(A^{\bullet}, \underline{\alpha}\right)+\delta \cdot \frac{1}{s !} \cdot \mu\left(A^{\bullet}, \underline{\alpha} ; \widehat{\varphi}\right)=P_{\alpha}\left(A^{\bullet}, \underline{\alpha}\right)+\delta \cdot v\left(A^{\bullet}, \underline{\alpha} ; \varphi\right)(\geq) 0
$$

holds.

Proposition 4.2.3. For the fixed input data $\varrho, s$, and $\alpha$ as above, there is a rational number $\delta_{\infty}>0$ such that for every $\delta>\delta_{\infty}$ and every singular $G$-bundle $(A, q, \tilde{\tau})$ with a GPS, the following conditions are equivalent:

(i) $(A, q, \tilde{\tau})$ is $(\alpha, \delta)$-(semi)stable.

(ii) $(A, \widetilde{\tau})$ is a principal $G$-bundle and, for every reduction $\beta$ of $(A, \widetilde{\tau})$ to a one-parameter subgroup $\lambda$ of $G$, one has

$$
P_{\alpha}\left(A_{\beta}^{\bullet}, \underline{\alpha}_{\beta}\right)(\geq) 0 .
$$

Proof. We apply Theorem 3.3.2. We first note that, by Corollary 4.1.2, $\widehat{\sim}$ is generically semistable if and only if the section $\tilde{\sigma}: \widetilde{X} \rightarrow \mathscr{H} o m\left(A, V^{\vee} \otimes \mathscr{O}_{\tilde{X}}\right) / / G$ associated with $\tilde{\tau}$ maps the generic point of $\widetilde{X}$ to $\mathscr{I}_{\operatorname{SOm}}\left(A, V^{\vee} \otimes \mathscr{O}_{\tilde{X}}\right) / G$. By Lemma 4.2.1 this means the same as $(A, \tilde{\tau})$ being a $G$-bundle. Finally, according to Proposition 4.2.2 the weighted filtrations $\left(A^{\bullet}, \underline{\alpha}\right)$ with $\mu\left(A^{\bullet}, \underline{\alpha}, \widehat{\varphi}\right)=0$ are precisely those of the form $\left(A_{\beta}^{\bullet}, \underline{\alpha}_{\beta}\right)$.

A family of singular G-bundles with a GPS parameterized by the scheme $S$ is a triple $\left(A_{S}, q_{S}, \tilde{\tau}_{S}\right)$ which consists of a vector bundle $A_{S}$ on $S \times \widetilde{X}$, a quotient map $q_{S}$ : $\bar{\pi}_{S \star}\left(A_{S \mid S_{N_{1}, N_{2}}}\right) \rightarrow R_{S}$ onto a vector bundle of rank $r$ on $S_{N}$, and a homomorphism

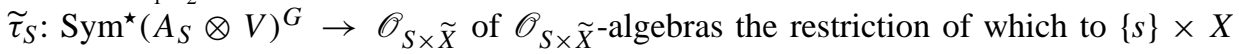
is non-trivial for every point $s \in S$. Recall that $\bar{\pi}_{S}$ denotes the projection morphism $S_{N_{1}, N_{2}} \rightarrow S_{N}$. Two such families $\left(A_{S}^{1}, q_{S}^{1}, \tilde{\tau}_{S}^{1}\right)$ and $\left(A_{S}^{2}, q_{S}^{2}, \widetilde{\tau}_{S}^{2}\right)$ are called equivalent if there is an isomorphism $\psi_{S}: A_{S}^{1} \rightarrow A_{S}^{2}$ such that

$$
q_{S}^{1}=q_{S}^{2} \circ \bar{\pi}_{S \star}\left(\psi_{S \mid S_{N_{1}, N_{2}}}\right) \quad \text { and } \quad \tilde{\tau}_{S}^{1}=\tilde{\tau}_{S}^{2} \circ \bar{\psi}_{S}
$$

with $\bar{\psi}_{S}: \operatorname{Sym}^{\star}\left(A_{S}^{1} \otimes V\right)^{G} \rightarrow \operatorname{Sym}^{\star}\left(A_{S}^{2} \otimes V\right)^{G}$ the isomorphism induced by $\psi_{S}$. The pullback of families of generalized parabolic bundles, introduced in Section 2.3 yields a pullback of families of singular $G$-bundles with a GPS, so that we may define the moduli functors

$$
\begin{aligned}
\underline{\operatorname{SPBGPS}}(\varrho)^{(\alpha, \delta)-(\mathrm{s}) \mathrm{s}}: \underline{\mathrm{Sch}} \underline{\mathbb{C}} & \rightarrow \frac{\text { Set, }}{S} \\
S & \left.\mapsto \begin{array}{l}
\text { equivalence classes of families of } \\
(\alpha, \delta) \text {-(semi)stable singular } \\
\text { principal bundles }\left(A_{S}, q_{S}, \widetilde{\tau}_{S}\right) \\
\text { with a GPS parameterized by } S
\end{array}\right\} .
\end{aligned}
$$

An intermediate step towards the main theorem is 
Theorem 4.2.4. There exist a projective scheme $\mathscr{S} \mathscr{P} \mathscr{B} \mathscr{G} \mathscr{P} \mathscr{S}(\varrho)^{(\alpha, \delta)-(\mathrm{s}) \mathrm{s}}$ and an open subscheme $\mathscr{S} \mathscr{P} \mathscr{B} \mathscr{G} \mathscr{P} \mathscr{S}(\varrho)^{(\alpha, \delta)-\mathrm{s}} \subset \mathscr{S} \mathscr{P} \mathscr{B} \mathscr{G} \mathscr{P} \mathscr{S}(\varrho)^{(\alpha, \delta) \text {-ss }}$ as well as natural transformations of functors

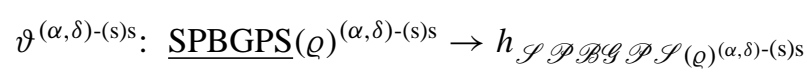

with the following properties:

1. For every $\mathscr{N}$ and every natural transformation $\vartheta^{\prime}: \underline{\operatorname{SPBGPS}}(\varrho)^{(\alpha, \delta) \text {-ss }} \rightarrow h_{\mathscr{N}}$, there is a unique morphism $\psi: \mathscr{S} \mathscr{P} \mathscr{B} \mathscr{G} \mathscr{P} \mathscr{S}(\varrho)^{(\alpha, \delta) \text {-ss }} \rightarrow \mathscr{N}$ with $\vartheta^{\prime}=h(\psi) \circ \vartheta^{(\alpha, \delta) \text {-ss }}$.

2. The scheme $\mathscr{S} \mathscr{P} \mathscr{B} \mathscr{G} \mathscr{P} \mathscr{S}(\varrho)^{(\alpha, \delta)-\mathrm{s}}$ is a coarse moduli space for the functor $\underline{\operatorname{SPBGPS}}(\varrho)^{(\alpha, \delta)-\mathrm{s}}$.

\subsection{Families of descending G-bundles}

The last notion which is missing is that of a family of descending principal $G$-bundles parameterized by a scheme $S$. First, let $\left(A_{S}, q_{S}, \tilde{\tau}_{S}\right)$ be a family of singular $G$-bundles with a GPS and $\mathscr{A}_{S}$ the associated family of torsion free sheaves on $S \times X$. As shown for the case $S=\{\star\}$ in Section 1.2, there is an associated homomorphism

$$
\tau_{S}^{\prime}: \operatorname{Sym}^{\star}\left(\mathscr{A}_{S} \otimes V\right)^{G} \rightarrow\left(\operatorname{id}_{S} \times \nu\right)_{\star} \mathscr{O}_{S \times \tilde{X}}
$$

of $\mathscr{O}_{S \times X}$-algebras. With the usual arguments, one checks

Lemma 4.3.1. Let $\left(A_{S}, q_{S}, \tilde{\tau}_{S}\right)$ be as above, $f: T \rightarrow S$ a morphism, and $\left(A_{T}, q_{T}, \tilde{\tau}_{T}\right)$ the pullback of the family $\left(A_{S}, q_{S}, \tilde{\tau}_{S}\right)$ via $f$. Then

$$
\tau_{T}^{\prime}=f^{\star}\left(\tau_{S}^{\prime}\right)
$$

Now, the family $\left(A_{S}, q_{S}, \tilde{\tau}_{S}\right)$ is called a family of descending principal G-bundles parameterized by the scheme $S$ if the image of the homomorphism $\tau_{S}^{\prime}$ lies in $\mathscr{O}_{S \times X}$.

Remark 4.3.2. This last condition is equivalent to the vanishing of

$$
\operatorname{Sym}^{\star}\left(\mathscr{A}_{S} \otimes V\right)^{G} \rightarrow\left(\left(\operatorname{id}_{S} \times v\right)_{\star} \mathscr{O}_{S \times \tilde{X}}\right) / \mathscr{O}_{S \times X} \stackrel{2.1 .1 / \sqrt[k]{2.1 .2}}{=} \pi_{X}^{\star}\left(\left(v_{\star} \mathscr{O}_{\tilde{X}}\right) / \mathscr{O}_{X}\right)
$$

Lemma 4.3.1 and Proposition 2.2.1 suggest that this is a reasonable condition.

\section{Singular $G$-bundles on nodal curves}

In this section, we explain the relationship with the problem of Nagaraj and Seshadri [13] and recent work of U. Bhosle [3]. 


\subsection{Descending $\mathrm{SL}_{r}(\mathbb{C})$-bundles}

The first interesting testing case for our results is the case of the semisimple group $\mathrm{SL}_{r}(\mathbb{C})$ together with its standard representation $\varrho: \mathrm{SL}_{r}(\mathbb{C}) \subset \mathrm{GL}_{r}(\mathbb{C})$. For any vector bundle $A$ on the curve $\widetilde{X}$, we have a canonical $\mathrm{GL}_{r}(\mathbb{C})$-equivariant isomorphism

$$
\mathscr{H} o m\left(A, \mathscr{O}_{\widetilde{X}}^{\oplus r}\right) / / \mathrm{SL}_{r}(\mathbb{C}) \rightarrow \mathscr{H} o m\left(\operatorname{det}(A), \mathscr{O}_{\widetilde{X}}\right),
$$

i.e., giving a non-trivial homomorphism $\tilde{\tau}: \operatorname{Sym}^{\star}(A \otimes V)^{\mathrm{SL}_{r}(\mathbb{C})} \rightarrow \mathscr{O}_{\widetilde{X}}$ is the same as giving a non-trivial homomorphism (= isomorphism, because $\operatorname{deg}(A) \stackrel{\mathbb{X}}{=} 0) \operatorname{det}(A) \rightarrow$ $\mathscr{O}_{\widetilde{X}}$. This implies that

$$
\mu\left(A^{\bullet}, \underline{\alpha} ; \widetilde{\tau}\right)=0
$$

for any weighted filtration $\left(A^{\bullet}, \underline{\alpha}\right)$ of $A$. Therefore, for any $\alpha \in(0,1) \cap \mathbb{Q}$, the condition of $\alpha$-(semi)stability on a descending $G$-bundle $(A, q, \widetilde{\tau})$ is the same as the condition of $\alpha$-(semi)stability on the generalized parabolic bundle $(A, q)$. Thus, we obtain a forgetful morphism

$$
\mathscr{M}(\varrho)^{\alpha-\mathrm{ss}} \rightarrow \mathscr{G} \mathscr{P} \mathscr{B}_{0 / r}^{\alpha-s s}
$$

and, for $\alpha$ close enough to one, also a forgetful morphism (Remark 3.1.1)

$$
\varphi: \mathscr{M}(\varrho)^{\alpha-s s} \rightarrow \mathscr{U}(r, 0) .
$$

Proposition 5.1.1. The set-theoretic image of the morphism $\varphi$ is the closed subset $\mathscr{U}_{\mathrm{SL}_{r}(\mathbb{C})}$.

Proof. Remarks 1.2.1 ii) and 3.1.1 imply that every point $u \in \mathscr{U}(r, 0)$ corresponding to a stable vector bundle $\mathscr{A}$ with trivial determinant lies in the image of $\varphi$. On the other hand, let $m \in \mathscr{M}(\varrho)^{\alpha-\text { ss }}$ be a point corresponding to a descending $G$-bundle $(A, q, \widetilde{\tau})$. Recall that we have an induced, non-trivial homomorphism of $\mathscr{O}_{X}$-algebras

$$
\tau: \operatorname{Sym}^{\star}\left(\mathscr{A}^{\oplus r}\right)^{\mathrm{SL}_{r}(\mathbb{C})} \rightarrow \mathscr{O}_{X}
$$

which corresponds to a section $\sigma: X \rightarrow \mathscr{H} o m\left(\mathscr{A}, \mathscr{O}_{X}^{\oplus r}\right) / / \mathrm{SL}_{r}(C)$. Note that, on the scheme $\mathscr{H}$ om $\left(\mathscr{A}, \mathscr{O}_{X}^{\oplus r}\right) \stackrel{\pi}{\rightarrow} X$, there is the tautological homomorphism $H: \pi^{\star}(\mathscr{A}) \rightarrow$ $\mathscr{O}_{\mathscr{H} \text { om }\left(\mathscr{A}, \mathscr{O}_{X}^{\oplus r}\right)}^{\oplus r}$. The homomorphism $\bigwedge^{r} H$ yields a morphism

$$
h: \mathscr{H} o m\left(\mathscr{A}, \mathscr{O}_{X}^{\oplus r}\right) \rightarrow \mathscr{H} o m\left(\bigwedge^{r} \mathscr{A}, \mathscr{O}_{X}\right) .
$$

Since $h$ is $\mathrm{SL}_{r}(\mathbb{C})$-invariant, it descends to a morphism

$$
\bar{h}: \mathscr{H} o m\left(\mathscr{A}, \mathscr{O}_{X}^{\oplus r}\right) / / \mathrm{SL}_{r}(\mathbb{C}) \rightarrow \mathscr{H} o m\left(\bigwedge^{r} \mathscr{A}, \mathscr{O}_{X}\right) .
$$

Thus, the homomorphism $\tau$ induces a homomorphism $\mathfrak{d}: \bigwedge^{r} \mathscr{A} \rightarrow \mathscr{O}_{X}$. Now, $\mathfrak{d}$ is ob-

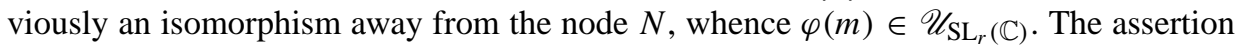
follows, because $\mathscr{U}_{\mathrm{SL}_{r}(\mathbb{C})}$ is the closure of the set of points $[\mathscr{A}] \in \mathscr{U}(r, 0)$ for which $\mathscr{A}$ is a stable vector bundle and $\operatorname{det}(\mathscr{A})$ is trivial ([22, Theorem 1]).

Therefore, we may equip $\mathscr{U}_{\mathrm{SL}(r)}$ with the structure of the scheme-theoretic image of the morphism $\varphi$. 


\subsection{A generalization to arbitrary semisimple groups}

A singular principal $G$-bundle on the nodal curve $X$ is a pair $(\mathscr{A}, \tau)$ which consists of a torsion free sheaf $\mathscr{A}$ of rank $r$ and degree zero on $X$ and a non-trivial homomorphism $\tau: \operatorname{Sym}^{\star}(\mathscr{A} \otimes V)^{G} \rightarrow \mathscr{O}_{X}$. Here, non-trivial means again that $\tau$ is not just the projection onto the degree zero component.

For a given singular $G$-bundle $(\mathscr{A}, \tau)$ we may choose an $s \gg 0$ such that the sum $\bigoplus_{i=1}^{s} \operatorname{Sym}^{i}(\mathscr{A} \otimes V)^{G}$ contains a set of generators for the algebra $\operatorname{Sym}^{\star}(\mathscr{A} \otimes V)^{G}$. For such an $s$, we obtain again an associated homomorphism

$$
\widehat{\varphi}:\left((\mathscr{A} \otimes V)^{\otimes s !}\right)^{\oplus N} \rightarrow \mathscr{O}_{X} .
$$

For a weighted filtration $\left(\mathscr{A}^{\bullet}, \underline{\alpha}\right)$ of $\mathscr{A}$, we set

$$
\begin{aligned}
\mu\left(\mathscr{A}^{\bullet}, \underline{\alpha} ; \widehat{\varphi}\right):=-\min \left\{\gamma_{j_{1}}+\cdots+\gamma_{j_{a}} \mid\left(j_{1}, \ldots, j_{a}\right) \in\{1, \ldots, s+1\}^{\times a},\right. \\
\left.\widehat{\varphi}_{\mid\left(\mathscr{A}_{j_{1}} \otimes \cdots \otimes \mathscr{A}_{j_{a}}\right) \oplus b} \not \equiv 0\right\}
\end{aligned}
$$

and

$$
\mu\left(\mathscr{A}^{\bullet}, \underline{\alpha} ; \tau\right):=\frac{1}{s !} \cdot \mu\left(\mathscr{A}^{\bullet}, \underline{\alpha} ; \widehat{\varphi}\right) .
$$

Given a positive rational number $\delta$, a singular $G$-bundle $(\mathscr{A}, \tau)$ is called $\delta$-(semi)stable if

$$
L\left(\mathscr{A}^{\bullet}, \underline{\alpha}\right)+\delta \cdot \mu\left(\mathscr{A}^{\bullet}, \underline{\alpha} ; \tau\right)(\geq) 0
$$

for every weighted filtration $\left(\mathscr{A}^{\bullet}, \underline{\alpha}\right)$ of $\mathscr{A}$.

Remark 5.2.1. (i) As in [16, Remark 3.6], one verifies that the quantity $\mu(\cdot, \cdot ; \tau)$ does not depend on the choice of $s$.

(ii) It is, in fact, sufficient to choose $s$ such that $\bigoplus_{i=1}^{s} \operatorname{Sym}^{i}(\mathscr{A} \otimes V)^{G}$ generates $\operatorname{Sym}^{\star}(\mathscr{A} \otimes V)^{G}$ over a non-empty open subset $U$. This follows again from the remarks in [16].

For a fixed $\delta \in \mathbb{Q}_{>0}$, we obtain the moduli functors $\underline{\mathrm{SPB}}(\varrho)^{\delta \text {-(s)s }}$ of $\delta$-(semi)stable singular $G$-bundles on $X$, and Bhosle proves in [3] that the moduli spaces $\mathscr{S} \mathscr{P} \mathscr{B}(\varrho)^{\delta \text {-(s)s }}$ for these functors do exist.

The problem with these concepts is that the theory of the instability flag à la Ramanan -Ramanathan, which is crucial to the investigations in [17] and [18], does not apply on the singular curve $X$. Therefore, we get no control over the open subset $U$ where a $\delta$ (semi)stable singular $G$-bundle $(\mathscr{A}, \tau)$ defines a true principal $G$-bundle ( $U$ might be even empty), nor do we obtain a satisfactory description of the concept of $\delta$-(semi)stability when $\delta$ gets large. For these reasons, we have worked, so far, on the smooth curve $\widetilde{X}$. Nevertheless, we may use our moduli spaces to define some interesting closed subschemes of Bhosle's moduli spaces.

In order to explain these ideas, we first remark that the concept of $(\alpha, \delta)$-(semi)stability and the numerical quantities involved may formally be defined for any positive rational number $\alpha$. In particular, we can speak of $(1, \delta)$-(semi)stability and define numbers such as 1-pardeg $(A)$, and $P_{1}\left(A^{\bullet}, \underline{\alpha}\right)$. 
Proposition 5.2.2. Let $(A, q, \widetilde{\tau})$ be a descending $G$-bundle and $(\mathscr{A}, \tau)$ the induced honest singular G-bundle on $X$.

(i) If $(\mathscr{A}, \tau)$ is $\delta$-(semi)stable, then $(A, q, \widetilde{\tau})$ is a $(1, \delta)-($ semi $)$ stable $G$-bundle with a GPS.

(ii) If $(A, q, \widetilde{\tau})$ is a $(1, \delta)$-(semi)stable $G$-bundle with a GPS, then $(\mathscr{A}, \tau)$ is a $\delta$-(semi) stable singular $G$-bundle.

Proof. The proof is essentially the same as the one of Proposition 4.2 in [2].

(i) Let $B$ be any saturated subsheaf of $A$. We define the saturated subsheaf $\mathscr{S}(B) \subset \mathscr{A}$ by means of the following commutative diagram:

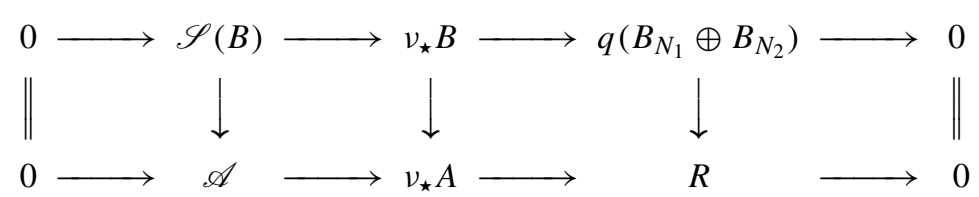

The Euler number of $\mathscr{S}(B)$ is

$$
\begin{aligned}
\chi(\mathscr{S}(B)) & =\chi(B)-\operatorname{dim} q\left(B_{N_{1}} \oplus B_{N_{2}}\right) \\
& =\operatorname{deg}(B)+\operatorname{rk}(B)(1-g(\widetilde{X}))-\operatorname{dim} q\left(B_{N_{1}} \oplus B_{N_{2}}\right) \\
& =\left(\operatorname{deg}(B)+\operatorname{rk} B-\operatorname{dim} q\left(B_{N_{1}} \oplus B_{N_{2}}\right)\right)+\operatorname{rk} \mathscr{S}(B)\left(1-p_{a}(X)\right),
\end{aligned}
$$

so that we conclude

$$
\begin{aligned}
\operatorname{deg}(\mathscr{A}) \mathrm{rk} \mathscr{S}(B) & -\operatorname{deg}(\mathscr{S}(B)) \mathrm{rk} \mathscr{A} \\
& =\operatorname{deg}(A) \mathrm{rk} B-\operatorname{deg}(\mathscr{S}(B)) \mathrm{rk} A \\
& =(\operatorname{deg}(A)-\operatorname{rk}(A)) \mathrm{rk} B-\left(\operatorname{deg}(B)-\operatorname{dim} q\left(B_{N_{1}} \oplus B_{N_{2}}\right)\right) \mathrm{rk} A \\
& =1-\operatorname{pardeg}(A) \cdot \operatorname{rk} B-1-\operatorname{pardeg}(B) \cdot \operatorname{rk} A .
\end{aligned}
$$

Given a weighted filtration $\left(A^{\bullet}, \underline{\alpha}\right)$, the above construction yields the weighted filtration $\left(\mathscr{A}^{\bullet}, \underline{\alpha}\right)$ with $\mathscr{A}_{j}:=\mathscr{S}\left(A_{j}\right), j=1, \ldots, s$, such that

$$
P_{1}\left(A^{\bullet}, \underline{\alpha}\right)=L\left(\mathscr{A}^{\bullet}, \underline{\alpha}\right) \text {. }
$$

Moreover, we clearly have

$$
\mu\left(A^{\bullet}, \underline{\alpha}, \widetilde{\tau}\right)=\mu\left(\mathscr{A}^{\bullet}, \underline{\alpha}, \tau\right)
$$

so that the first assertion is established.

(ii) To prove the second part of the proposition, we have to explain that every saturated subsheaf $\mathscr{B} \subsetneq \mathscr{A}$ is of the form $\mathscr{S}(B)$ for an appropriate subbundle $B$ of $A$. This is indeed the case for the subbundle $B$ generated by the image of the homomorphism

$$
\varphi: v^{\star} \mathscr{B} \rightarrow v^{\star} \mathscr{A} \rightarrow v^{\star} v_{\star} A \rightarrow A .
$$

The other ingredient we need is the following. 
Proposition 5.2.3. There is a number $\varepsilon>0$, such that for any $\alpha \in(1-\varepsilon, 1) \cap \mathbb{Q}$, any integral semistability parameter $\delta$, and any singular $G$-bundle $(A, q, \widetilde{\tau})$ with a GPS, we have:

(i) If $(A, q, \widetilde{\tau})$ is $(\alpha, \delta)$-semistable, then it is $(1, \delta)$-semistable.

(ii) If $(A, q, \widetilde{\tau})$ is $(1, \delta)$-stable, then it is $(\alpha, \delta)$-stable.

Proof. We remind the reader that there is a finite set $\mathscr{T}$, depending only on the $\operatorname{SL}(V)$ action on $\mathbb{P}\left(V \otimes \mathbb{C}^{r}\right) / / G$, such that the condition of $(\alpha, \delta)$-semistability for a singular $G$-bundle $(A, q, \widetilde{\tau})$ with a GPS has to be checked only against those weighted filtrations $\left(A^{\bullet}, \underline{\alpha}\right)$ of $A$ for which $\left(\mathrm{rk} A_{1}, \ldots, \mathrm{rk} A_{s}, \alpha_{1}, \ldots, \alpha_{s}\right) \in \mathscr{T}$ (Proposition 3.3.5). We may find a natural number $n$ such that

$$
P_{1}\left(A^{\bullet}, \underline{\alpha}\right)+\delta \cdot \mu\left(A^{\bullet}, \underline{\alpha} ; \widetilde{\tau}\right) \in \mathbb{Z}[1 / n]
$$

for any such weighted filtration. For a generalized parabolic bundle $(A, q)$ and a weighted filtration $\left(A^{\bullet}, \underline{\alpha}\right)$, we have

$$
\begin{aligned}
\left|P_{1}\left(A^{\bullet}, \underline{\alpha}\right)-P_{\alpha}\left(A^{\bullet}, \underline{\alpha}\right)\right| & <\varepsilon \cdot \sum_{j=1}^{s} \alpha_{j} \operatorname{rk} A_{j} \cdot\left(\operatorname{rk} A-\operatorname{dim} q\left(A_{j \mid N_{1}} \oplus A_{j \mid N_{2}}\right)\right) \\
& \leq \varepsilon \cdot \operatorname{rk} A \cdot \sum_{j=1}^{s} \alpha_{j} \operatorname{rk} A_{j} .
\end{aligned}
$$

We may choose $\varepsilon$ so small that the right hand side of the above inequality is strictly smaller than $1 / n$. For such an $\varepsilon$, the assertion is clear.

Now, let $\left(A_{S}, q_{S}, \widetilde{\tau}_{S}\right)$ be a family of descending $G$-bundles parameterized by the scheme $S$. Then there is an associated family $\left(\mathscr{A}_{S}, \tau_{S}\right)$ of (honest) singular $G$-bundles (on $X$ ) parameterized by $S$ (see Section 4.3). Let $\varepsilon$ be as in Proposition 5.2.3. Choose $\alpha \in(1-\varepsilon, 1) \cap \mathbb{Q}$ and $\delta \in \mathbb{Z}_{>0}$ so large that the conclusion of Proposition 4.2.3 holds. Remark 4.3.1. Proposition 5.2.2, and Proposition 5.2.3 imply that the assignment $\left(A_{S}, q_{S}, \widetilde{\tau}_{S}\right) \mapsto\left(\mathscr{A}_{S}, \tau_{S}\right)$ induces a natural transformation

$$
\underline{\mathrm{M}}(\varrho)^{\alpha-\mathrm{s}) \mathrm{s}} \rightarrow \underline{\mathrm{SPB}}(\varrho)^{\delta-(\mathrm{s}) \mathrm{s}}
$$

and, thus, a morphism

$$
\mathscr{M}(\varrho)^{\alpha-(\mathrm{s}) \mathrm{s}} \rightarrow \mathscr{S} \mathscr{P} \mathscr{B}(\varrho)^{\delta \text {-(s)s }}
$$

between the corresponding moduli spaces. We define $\mathscr{M}_{X}(\varrho) \subset \mathscr{S} \mathscr{P} \mathscr{B}(\varrho)^{\delta \text {-ss }}$ as the scheme-theoretic image of that morphism. This space is a good candidate for (at least the point set of) a compactified moduli space of stable $G$-bundles on $X$. Note that the above considerations demonstrate that $\mathscr{M}_{X}(\varrho)$ consists of semistable honest singular $G$-bundles on $X$ and that, indeed, every stable honest singular $G$-bundle on $X$ lies in $\mathscr{M}_{X}(\varrho)$. 


\subsection{An open problem}

The main problem with the results we have established in this paper is the lack of compatibility of the moduli spaces with degenerations. In fact, given a family $\mathfrak{X} \rightarrow C$ over, say, $C=\operatorname{Spec} \mathbb{C}[t \rrbracket$, such that the generic fibre is smooth and the fibre over 0 is a nodal curve $X$, one would like to have a relative moduli space $\mathfrak{M}_{G} \rightarrow C$ such that the generic fibre is the usual moduli space of semistable $G$-bundles and such that the fibre over 0 is the moduli space we have constructed. This seems impossible in our approach. What is, however, possible is the construction of a family $\mathfrak{s}: \mathfrak{S}(\varrho)^{\delta \text {-ss }} \rightarrow C$ such that over the generic fibre we find our moduli space of $\delta$-semistable singular $G$-bundles from [16] and over 0 we find Bhosle's moduli space $\mathscr{S} \mathscr{P} \mathscr{B}^{\delta \text {-ss }}$. By the results of [17], the generic fibre is the usual moduli space of semistable $G$-bundles provided that $\delta$ is large enough. So, in the wake of Sun's results [22], we suggest the following

Problem. Let $\mathfrak{m}: \widehat{\mathfrak{M}}_{G} \rightarrow C$ be the closure of $\mathfrak{s}^{-1}(C \backslash\{0\})$. Does the fibre $\mathfrak{m}^{-1}(0)$ with its induced reduced scheme structure equal $\mathscr{M}_{X}(\varrho)$ with its induced reduced scheme structure?

\section{The proofs}

In this section, we discuss the construction of the various moduli spaces we have encountered so far. The main ideas are derived from our previous papers [15] and [16] and Bhosle's theory of generalized parabolic bundles [2].

\subsection{Proof of Theorem 3.1 .2}

Boundedness. Recall that a family $\mathfrak{B}$ of isomorphy classes $[A]$ of vector bundles on $\widetilde{X}$ of, say, degree $d$ and rank $r$ is said to be bounded if there exist a scheme $S$ of finite type over $\mathbb{C}$ and a vector bundle $A_{S}$ on $S \times \widetilde{X}$ such that for every vector bundle $A$ on $\widetilde{X}$ with $[A] \in \mathfrak{B}$, there exists a point $s \in S$ with $A \cong A_{S \mid\{s\} \times \widetilde{X}}$. By the semicontinuity theorem and easy facts on vector bundles on curves, this is equivalent to the fact that there is a constant $C$ such that $\mu(B) \leq d / r+C$ for every bundle $A$ with $[A] \in \mathfrak{B}$ and every subbundle $0 \subsetneq B \subsetneq A$.

Proposition 6.1.1. There is a non-negative constant $C$, depending only on $r$, $a$, and $\delta$, such that for every $(\alpha, \delta)$-semistable generalized parabolic bundle $(A, q, \varphi)$ with a decoration of type $(a, b, c ; L)$ and every non-trivial proper subbundle $B$ of $A$,

$$
\mu(B) \leq \frac{d}{r}+C .
$$

Proof. Let $0 \subsetneq B \subsetneq A$ be any subbundle. Lemma 1.8(i) in [15] shows

$$
\mu_{a, b, c}(0 \subsetneq B \subsetneq A,(1) ; \varphi) \leq a(r-1),
$$


so that $(\alpha, \delta)$-semistability gives

$$
\begin{aligned}
& d \mathrm{rk} B-(\operatorname{deg}(B)-r) r+\delta \cdot a \cdot(r-1) \\
& \quad \geq(d-\alpha \cdot r) \mathrm{rk} B-\left(\operatorname{deg}(B)-\alpha \cdot \operatorname{dim} q\left(B_{N_{1}} \oplus B_{N_{2}}\right)\right) r+\delta \cdot a \cdot(r-1) \\
& \quad=\alpha-\operatorname{pardeg}(A) \mathrm{rk} B-\alpha-\operatorname{pardeg}(B) r+\delta \cdot a \cdot(r-1) \\
& \quad \geq \alpha-\operatorname{pardeg}(A) \mathrm{rk} B-\alpha-\operatorname{pardeg}(B) r+\delta \cdot \mu_{a, b, c}(0 \subsetneq B \subsetneq A,(1) ; \varphi) \geq 0 .
\end{aligned}
$$

We see that

$$
\mu(B) \leq \frac{d}{r}+\frac{\delta \cdot a \cdot(r-1)+r^{2}}{r \cdot \operatorname{rk}(B)} \leq \frac{d}{r}+\frac{\delta \cdot a \cdot(r-1)}{r}+r,
$$

so that the assertion holds with $C=r+\delta a(r-1) / r$.

Construction of the parameter space. Recall that, for a scheme $S$ of finite type over $\mathbb{C}$, a family of generalized parabolic bundles with a decoration of type $(a, b, c ; L)$ parameterized by $S$ is a quadruple $\left(A_{S}, q_{S}, \mathfrak{N}_{S}, \varphi_{S}\right)$, where $\left(A_{S}, q_{S}\right)$ is a family of generalized parabolic bundles, $\mathfrak{N}_{S}$ is a line bundle on $S$, and $\varphi_{S}:\left(A_{S}^{\otimes a}\right)^{\oplus b} \rightarrow \operatorname{det}\left(A_{S}\right)^{\otimes c} \otimes \pi_{\widetilde{X}}^{\star} L \otimes$ $\pi_{S}^{\star} \mathfrak{N}_{S}$ is a homomorphism such that $\varphi_{S \mid\{s\} \times \widetilde{X}} \not \equiv 0$ for all $s \in S$.

We choose a point $x_{0} \in \widetilde{X}$ and write $\mathscr{O}_{\widetilde{X}}(1)$ for $\mathscr{O}_{\widetilde{X}}\left(x_{0}\right)$. By 6.1.1, we may choose an integer $n_{0}$ such that for every $n \geq n_{0}$ and every $(\alpha, \delta)$-semistable generalized parabolic bundle $(A, q, \varphi)$ with a decoration of type $(a, b, c ; L)$ :

- $H^{1}(A(n))=0$ and $A(n)$ is globally generated,

- $H^{1}(\operatorname{det}(A)(r n))=0$ and $\operatorname{det}(A)(r n)$ is globally generated,

- $H^{1}\left(\operatorname{det}(A)^{\otimes c} \otimes L \otimes \mathscr{O}_{\widetilde{X}}(n a)\right)=0$ and $\operatorname{det}(A)^{\otimes c} \otimes L \otimes \mathscr{O}_{\widetilde{X}}(n a)$ is globally generated.

Choose some $n \geq n_{0}$ and set $p:=d+r n+r(1-g)$. Let $U$ be a complex vector space of dimension $p$. We define $\mathfrak{Q}^{0}$ as the quasi-projective scheme parameterizing equivalence classes of quotients $\mathfrak{q}: U \otimes \mathscr{O}_{X}(-n) \rightarrow A$, where $A$ is a vector bundle of rank $r$ and degree $d$ on $\widetilde{X}$ and $H^{0}(q(n))$ is an isomorphism. On $\mathfrak{Q}^{0} \times \widetilde{X}$, we have the universal quotient

$$
\mathfrak{q}_{\mathfrak{Q}^{0}}: U \otimes \pi_{\widetilde{X}}^{\star} \mathscr{O}_{\widetilde{X}}(-n) \rightarrow A_{\mathfrak{Q}^{0}}
$$

Define $U_{a, b}:=\left(U^{\otimes a}\right)^{\oplus b}$. Our assumptions imply that the sheaf

$$
\mathscr{H} \text { om }\left(U_{a, b} \otimes \mathscr{O}_{\mathfrak{Q}^{0}}, \pi_{\mathfrak{Q}^{0}}\left(\operatorname{det}\left(A_{\mathfrak{Q}^{0}}\right)^{\otimes c} \otimes \pi_{\widetilde{X}}^{\star} L \otimes \pi_{\widetilde{X}}^{\star} \mathscr{O}_{\widetilde{X}}(n a)\right)\right)
$$

is locally free. We call this sheaf $\mathscr{H}$ and set $\mathfrak{H}:=\mathbb{P}\left(\mathscr{H}^{\vee}\right)$. We let

$$
\mathfrak{q}_{\mathfrak{H}}: U \otimes \pi_{\widetilde{X}}^{\star} \mathscr{O}_{\widetilde{X}}(-n) \rightarrow A_{\mathfrak{H}}
$$

be the pullback of $\mathfrak{q}_{\mathfrak{Q}^{0}}$ to $\mathfrak{H} \times \widetilde{X}$. Now, on $\mathfrak{H} \times X$, there is the tautological homomorphism

$$
s_{\mathfrak{H}}: U_{a, b} \otimes \mathscr{O}_{\mathfrak{H}} \rightarrow \operatorname{det}\left(A_{\mathfrak{H}}\right)^{\otimes c} \otimes \pi_{\widetilde{X}}^{\star} L \otimes \pi_{\widetilde{X}}^{\star} \mathscr{O}_{\widetilde{X}}(n a) \otimes \pi_{\mathfrak{H}}^{\star} \mathscr{O}_{\mathfrak{H}}(1)
$$


According to Proposition 2.2.1, we define $\mathfrak{S}$ as the closed subscheme which is characterized by the condition that $s_{\mathfrak{H}} \otimes \pi_{\widetilde{X}}^{\star} \operatorname{id}_{\mathscr{O}_{\widetilde{X}}(-n a)}$ vanish on

$$
\operatorname{ker}\left(U_{a, b} \otimes \pi_{\widetilde{X}}^{\star} \mathscr{O}_{\widetilde{X}}(-n a) \rightarrow\left(A_{\mathfrak{H}}^{\otimes a}\right)^{\oplus b}\right)
$$

Let

$$
\mathfrak{q}_{\mathfrak{S}}: U \otimes \pi_{\widetilde{X}}^{\star} \mathscr{O}_{\widetilde{X}}(-n) \rightarrow A_{\mathfrak{S}}
$$

be the restriction of $\mathfrak{q}_{\mathfrak{H}}$ to $\mathfrak{S} \times \widetilde{X}$. By definition of $\mathfrak{S}$, the section $s_{\mathfrak{H}}$ factorizes over a homomorphism

$$
\varphi_{\mathfrak{S}}:\left(A_{\mathfrak{S}}^{\otimes a}\right)^{\oplus b} \rightarrow \operatorname{det}\left(A_{\mathfrak{S}}\right)^{\otimes c} \otimes \pi_{\widetilde{X}}^{\star} L \otimes \pi_{\mathfrak{S}}^{\star} \mathfrak{N}_{\mathfrak{S}}
$$

Here, $\mathfrak{N}_{\mathfrak{S}}$ is the restriction of $\mathscr{O}_{\mathfrak{H}}(1)$ to $\mathfrak{S}$. The space $\mathfrak{S}$ comes equipped with a family $\left(A_{\mathfrak{S}}, \mathfrak{N}_{\mathfrak{S}}, \varphi_{\mathfrak{S}}\right)$. To incorporate the parabolic structure as well, we let $\mathfrak{G}$ be the Graßmannian of $r$-dimensional quotients of $U$. Let $q_{\mathfrak{G}}: U \otimes \mathscr{O}_{\mathfrak{G}} \rightarrow R_{\mathfrak{G}}$ be the universal quotient and pull it back to $\mathfrak{S} \times \mathfrak{G}$ in order to get

$$
q_{\mathfrak{S} \times \mathfrak{G}}: U \otimes \mathscr{O}_{\mathfrak{S} \times \mathfrak{G}} \rightarrow R_{\mathfrak{S} \times \mathfrak{G}}
$$

We define $\mathfrak{q}_{\mathfrak{S} \times \mathfrak{G}}, A_{\mathfrak{S} \times \mathfrak{G}}, \mathfrak{N}_{\mathfrak{S} \times \mathfrak{G}}$, and $\varphi_{\mathfrak{S} \times \mathfrak{G}}$ as the pullbacks of the objects $\mathfrak{q}_{\mathfrak{S}}, A_{\mathfrak{S}}$, $\mathfrak{N}_{\mathfrak{S}}$, and $\varphi_{\mathfrak{S}}$ on $\mathfrak{S}$ and $\mathfrak{S} \times \widetilde{X}$ to $\mathfrak{S} \times \mathfrak{G}$ and $\mathfrak{S} \times \mathfrak{G} \times \widetilde{X}$, respectively. On the scheme $\mathfrak{S} \times \mathfrak{G}=\mathfrak{S} \times \mathfrak{G} \times\{N\}$, we have the quotient

$$
\mathfrak{q}_{N}: U \otimes \mathscr{O}_{\mathfrak{S} \times \mathfrak{G} \rightarrow \bar{\pi}}(\mathfrak{S} \times \mathfrak{G})_{N_{1}, N_{2}}{ }\left(A_{\mathfrak{S} \times \mathfrak{G} \mid(\mathfrak{S} \times \mathfrak{G})_{N_{1}, N_{2}}}\right)
$$

obtained by first restricting $\mathfrak{q} \mathfrak{S} \times \mathfrak{G}$ to $\mathfrak{S} \times \mathfrak{G} \times\left\{N_{1}, N_{2}\right\}$ and then projecting to $\mathfrak{S} \times \mathfrak{G} \times\{N\}$. We define the closed subscheme $\mathfrak{T}$ as the zero locus of the induced vector bundle map $\operatorname{ker}\left(\mathfrak{q}_{N}\right) \rightarrow R_{\mathfrak{S} \times \mathfrak{G}}$, so that the restriction of $q_{\mathfrak{S} \times \mathfrak{G}}$ to $\mathfrak{T}$ factorizes over a quotient

$$
q_{\mathfrak{T}}: \bar{\pi}_{(\mathfrak{S} \times \mathfrak{G})_{N_{1}, N_{2}}}\left(A_{\mathfrak{S} \times \mathfrak{G} \mid(\mathfrak{S} \times \mathfrak{G})_{N_{1}, N_{2}}}\right) \mid \mathfrak{T}=\bar{\pi}_{\mathfrak{T}_{N_{1}, N_{2}}{ }}\left(A \mathfrak{T} \mid \mathfrak{T}_{N_{1}, N_{2}}\right) \rightarrow R_{\mathfrak{T}}:=R_{\mathfrak{S} \times \mathfrak{G} \mid \mathfrak{T} .}
$$

Let $A_{\mathfrak{T}}$ and $\varphi_{\mathfrak{T}}$ be the restrictions of $A_{\mathfrak{S} \times \mathfrak{G}}$ and $\varphi_{\mathfrak{S} \times \mathfrak{G}}$ to $\mathfrak{T} \times \tilde{X}$, and set $\mathfrak{N}_{\mathfrak{T}}:=\mathfrak{N}_{\mathfrak{S} \times \mathfrak{G} \mid \mathfrak{T}}$. We call $\left(A_{\mathfrak{T}}, q_{\mathfrak{T}}, \mathfrak{N}_{\mathfrak{T}}, \varphi_{\mathfrak{T}}\right)$ the universal family. This is justified by

Proposition 6.1.2 (Local universal property). Let $S$ be a scheme of finite type over $\mathbb{C}$, and $\left(A_{S}, q_{S}, \mathfrak{N}_{S}, \varphi_{S}\right)$ a family of $(\alpha, \delta)$-semistable generalized parabolic bundles with a decoration of type $(a, b, c ; L)$ parameterized by $S$. Then there exist an open covering $S_{i}, i \in I$, of $S$, and morphisms $\beta_{i}: S_{i} \rightarrow \mathfrak{T}, i \in I$, such that the restriction of the family $\left(A_{S}, q_{S}, \mathfrak{N}_{S}, \varphi_{S}\right)$ to $S_{i} \times \widetilde{X}$ is equivalent to the pullback of $\left(A_{\mathfrak{T}}, q_{\mathfrak{T}}, \mathfrak{N}_{\mathfrak{T}}, \varphi_{\mathfrak{T}}\right)$ via $\beta_{i} \times \mathrm{id}_{\tilde{X}}$, for all $i \in I$.

Proof. This is standard (see, e.g., [15, Proposition 2.8]). 
The group action. We have natural actions of the group $\operatorname{SL}(U)$ on the schemes $\mathfrak{Q}^{0}, \mathfrak{H}$, and $\mathfrak{G}$. These actions induce an action

$$
\Gamma: \operatorname{SL}(U) \times \mathfrak{T} \rightarrow \mathfrak{T}
$$

of $\operatorname{SL}(U)$ on the parameter scheme $\mathfrak{T}$.

Proposition 6.1.3. Let $S$ be a scheme of finite type over $\mathbb{C}$ and $\beta_{1,2}: S \rightarrow \mathfrak{T}$ two morphisms such that the pullbacks of $\left(A_{\mathfrak{T}}, q_{\mathfrak{T}}, \mathfrak{N}_{\mathfrak{T}}, \varphi_{\mathfrak{T}}\right)$ via $\beta_{1} \times \mathrm{id}_{\tilde{X}}$ and $\beta_{2} \times \mathrm{id}_{\tilde{X}}$ are equivalent. Then there exist an étale covering $\eta: T \rightarrow S$ and a morphism $\Xi: T \rightarrow \operatorname{SL}(U)$ such that the morphism $\beta_{2} \circ \eta: T \rightarrow \mathfrak{T}$ equals

$$
T \stackrel{\Xi \times\left(\beta_{1} \circ \eta\right)}{\rightarrow} \operatorname{SL}(U) \times \mathfrak{T} \stackrel{\Gamma}{\rightarrow} \mathfrak{T}
$$

Proof. This can be easily adapted from [15, proof of Proposition 2.10].

The Gieseker space and map. Choose a Poincaré sheaf $\mathscr{P}$ on $\mathrm{Jac}^{d} \times \tilde{X}$. By our assumptions on $n$, the sheaf

$$
\mathscr{G}_{1}:=\mathscr{H} o m\left(\bigwedge^{r} U \otimes \mathscr{O}_{\mathrm{Jac}^{d}}, \pi_{\mathrm{Jac}^{d} \star}\left(\mathscr{P} \otimes \pi_{\widetilde{X}}^{\star} \mathscr{O}_{\widetilde{X}}(r n)\right)\right)
$$

is locally free. We set $\mathbb{G}_{1}:=\mathbb{P}\left(\mathscr{G}_{1}^{\vee}\right)$. By replacing $\mathscr{P}$ with $\mathscr{P} \otimes \pi_{\mathrm{Jac}^{d}}^{\star}$ (sufficiently ample), we may assume that $\mathscr{O}_{\mathbb{G}_{1}}(1)$ be very ample. Let $\mathfrak{d}: \mathfrak{T} \rightarrow \mathrm{Jac}^{d}$ be the morphism associated with $\bigwedge^{r} A_{\mathfrak{T}}$, and let $\mathfrak{A}_{\mathfrak{T}}$ be a line bundle on $\mathfrak{T}$ with $\bigwedge^{r} A_{\mathfrak{T}} \cong\left(\mathfrak{d} \times \operatorname{id}_{\widetilde{X}}\right)^{\star} \mathscr{P} \otimes \pi_{\mathfrak{T}}^{\star} \mathfrak{A}_{\mathfrak{T}}$. Then

$$
\bigwedge^{r}\left(\mathfrak{q}_{\mathfrak{T}} \otimes \operatorname{id}_{\pi_{\widetilde{X}}^{\star} \mathscr{O}_{\widetilde{X}}(n)}\right): \bigwedge^{r} U \otimes \mathscr{O}_{\mathfrak{T}} \rightarrow\left(\mathfrak{d} \times \operatorname{id}_{\widetilde{X}}\right)^{\star} \mathscr{P} \otimes \pi_{\widetilde{X}}^{\star} \mathscr{O}_{\widetilde{X}}(r n) \otimes \pi_{\mathfrak{T}^{\prime}} \mathfrak{A}_{\mathfrak{T}}
$$

defines a morphism $\iota_{1}: \mathfrak{T} \rightarrow \mathbb{G}_{1}$ with $\iota_{1}^{\star} \mathscr{O}_{\mathbb{G}_{1}}(1)=\mathfrak{A}_{\mathfrak{T}}$. The sheaf

$$
\mathscr{G}_{2}:=\mathscr{H} \text { om }\left(U_{a, c} \otimes \mathscr{O}_{\mathrm{Jac}^{d}}, \pi_{\mathrm{Jac}^{d} \star}\left(\mathscr{P} \otimes c \otimes \pi_{\widetilde{X}}^{\star} L \otimes \pi_{\widetilde{X}}^{\star} \mathscr{O}_{\widetilde{X}}(n a)\right)\right)
$$

on $\mathrm{Jac}^{d}$ is also locally free. Set $\mathbb{G}_{2}:=\mathbb{P}\left(\mathscr{G}_{2}^{\vee}\right)$. It is clear that we can assume $\mathscr{O}_{\mathbb{G}_{2}}(1)$ to be very ample as well. The homomorphism

$$
\begin{aligned}
U_{a, b} \otimes \mathscr{O}_{\mathfrak{T}} \rightarrow\left(A_{\mathfrak{T}}^{\otimes a}\right)^{\oplus b} \otimes \pi_{\widetilde{X}}^{\star} \mathscr{O}_{\widetilde{X}}(n a) \\
\quad \rightarrow\left(\mathfrak{d} \times \operatorname{id}_{\widetilde{X}}\right)^{\star} \mathscr{P} \otimes c \otimes \pi_{\widetilde{X}}^{\star} L \otimes \pi_{\widetilde{X}}^{\star} \mathscr{O}_{\widetilde{X}}(n a) \otimes \pi_{\mathfrak{T}}^{\star}\left(\mathfrak{A}_{\mathfrak{T}}^{\otimes c} \otimes \mathfrak{N}_{\mathfrak{T}}\right)
\end{aligned}
$$

provides a morphism $\iota_{2}: \mathfrak{T} \rightarrow \mathbb{G}_{2}$ with $\iota_{2}^{\star} \mathscr{O}_{\mathbb{G}_{2}}(1)=\mathfrak{A}_{\mathfrak{T}}^{\otimes c} \otimes \mathfrak{N}_{\mathfrak{T}}$. Finally, we have the morphism $\iota_{3}: \mathfrak{T} \rightarrow \mathfrak{G}$ from the construction of $\mathfrak{T}$. Altogether, setting $\mathbb{G}:=\mathbb{G}_{1} \times \mathbb{G}_{2} \times \mathfrak{G}$ and $\iota:=\iota_{1} \times \iota_{2} \times \iota_{3}$, we have an injective and $\operatorname{SL}(U)$-equivariant morphism

$$
\iota: \mathfrak{T} \rightarrow \mathbb{G} .
$$

Linearize the $\operatorname{SL}(U)$-action on $\mathbb{G}$ in $\mathscr{O}_{\mathbb{G}}(p-a \delta-r \alpha, r \delta, r \alpha)$ and denote by $\mathbb{G}^{(\mathrm{s}) \mathrm{s}}$ the sets of points in $\mathbb{G}$ which are $\operatorname{SL}(U)$-(semi)stable with respect to the given linearization. 
Theorem 6.1.4. For $n$ large enough, the following two properties hold true:

(i) The preimages $\iota^{-1}\left(\mathbb{G}^{(\mathrm{s}) \mathrm{s}}\right)$ consist exactly of those points $t \in \mathfrak{T}$ for which $\left(A_{t}, q_{t}, \varphi_{t}\right)$, the restriction of the universal family to $\{t\} \times \widetilde{X}$, is an $(\alpha, \delta)$-(semi)stable generalized parabolic bundle with a decoration of type $(a, b, c ; L)$.

(ii) The restricted morphism $\iota_{\iota^{-1}\left(\mathbb{G}^{\mathrm{ss}}\right)}: \iota^{-1}\left(\mathbb{G}^{\mathrm{ss}}\right) \rightarrow \mathbb{G}^{\mathrm{ss}}$ is proper.

If we set $\mathfrak{T}^{(\alpha, \delta)-(\mathrm{s}) \mathrm{s}}:=\iota^{-1}\left(\mathbb{G}^{(\mathrm{s}) \mathrm{s}}\right)$, the above theorem implies that

$$
\mathscr{D}:=\mathscr{D} \mathscr{G} \mathscr{P}_{d / r / a / b / c / L}^{(\alpha, \delta)-\mathrm{s}}:=\mathfrak{T}^{(\alpha, \delta) \text {-ss }} / / \operatorname{SL}(U)
$$

exists as a projective scheme. Propositions 6.1.2 and 6.1 .3 show that there is a natural transformation of the functor $\underline{\mathrm{DGPB}^{2}} d / r / a / b / c / L$ into the functor of points of $\mathscr{D}$. The universal property (Theorem 3.1.2 $\mathrm{i}$ )) of this transformation is then a consequence of the universal property of the categorical quotient. Likewise, one establishes 3.1.2 (ii) for the open subscheme

$$
\mathscr{D} \mathscr{G} \mathscr{P}_{d / r / a / b / c / L}^{(\alpha, \delta)-\mathrm{s}}:=\mathfrak{T}^{(\alpha, \delta)-\mathrm{s}} / \operatorname{SL}(U) .
$$

Proof of Theorem 6.1.4(i): A sample computation. The proof of Theorem 6.1.4 (i) may be carried out along the lines of the proof of Theorem 2.11 in [15], basically by replacing the degree with the $\alpha$-parabolic degree everywhere. Therefore, we do not present the whole proof here, but only a sample computation which demonstrates, in particular, that we have chosen the correct linearization on the Gieseker space $\mathbb{G}$.

We use the notation of [15] for the sample computation. We assume that we are given a point $t \in \iota^{-1}\left(\mathbb{G}^{(\mathrm{s}) \mathrm{s}}\right)$. This yields the decorated GPB $\left(A_{t}, q_{t}, \varphi_{t}\right)$. We will check the condition of $(\alpha, \delta)$-(semi)stability for all weighted filtrations $\left(A^{\bullet}, \underline{\alpha}\right)$ of $A$ for which $A_{j}(n)$ is globally generated and $H^{1}\left(A_{j}(n)\right)=\{0\}, j=1, \ldots, s$.

Remark 6.1.5. It is enough to consider such weighted filtrations because of the following observations:

1. The set of torsion free sheaves of the form $A_{t}$ with $t \in \iota^{-1}\left(\mathbb{G}^{\mathrm{ss}}\right)$ for some $n \geq n_{0}$ (needed to perform the construction) is also bounded (see [15, p. 188]).

2. Given any bounded family $\mathfrak{B}$ of vector bundles of rank $r$ and degree $d$ on $\widetilde{X}$, the condition of $(\alpha, \delta)$-(semi)stability for decorated GPBs $(A, q, \varphi)$ with $[A] \in \mathfrak{B}$ has to be tested only against weighted filtrations $\left(A^{\bullet}, \underline{\alpha}\right)$ satisfying the above extra assumptions. This is an easy consequence of Proposition 3.3.5 and general facts on bounded families (compare [18, Section 3.4]).

From the filtration $A^{\bullet}$ of $A_{t}$ and the quotient $\mathfrak{q}_{t}: U \otimes \mathscr{O}_{\widetilde{X}}(-n) \rightarrow A_{t}$, we get the flag $U^{\bullet}$ in $U$ with $U_{j}:=\left(H^{0}\left(\mathfrak{q}_{t}(n)\right)\right)^{-1}\left(H^{0}\left(A_{j}(n)\right)\right), j=1, \ldots, s$. We choose a one-parameter subgroup $\lambda: \mathbb{C}^{\star} \rightarrow \operatorname{SL}(U)$ the weighted flag of which is just $\left(U^{\bullet}, \underline{\alpha}\right)$. By assumption, writing $t=\left(t_{1}, t_{2}, t_{3}\right)$,

$$
\begin{aligned}
0 & (\leq) \mu(\lambda, \iota(t)) \\
& =(p-a \delta-r \alpha) \cdot \mu_{\mathbb{G}_{1}}\left(\lambda, t_{1}\right)+r \delta \cdot \mu_{\mathbb{G}_{2}}\left(\lambda, t_{2}\right)+r \alpha \cdot \mu_{\mathbb{G}_{3}}\left(\lambda, t_{3}\right)
\end{aligned}
$$




$$
\begin{aligned}
= & (p-a \delta-r \alpha) \cdot \sum_{j=1}^{s} \alpha_{j}\left(p \operatorname{rk} A_{j}-h^{0}\left(A_{j}(n)\right) r\right) \\
& +r \delta \cdot \sum_{j=1}^{s} \alpha_{j}\left(v_{j}\left(\iota_{0}\right) \cdot p-a \cdot h^{0}\left(A_{j}(n)\right)\right) \\
& +r \alpha \cdot \sum_{j=1}^{s} \alpha_{j}\left(p \operatorname{dim} q_{t}\left(A_{j \mid N_{1}} \oplus A_{j \mid N_{2}}\right)-r h^{0}\left(A_{j}(n)\right)\right) \\
= & \sum_{j=1}^{s} \alpha_{j}\left(p^{2} \operatorname{rk} A_{j}-p a \delta \operatorname{rk} A_{j}-p r \alpha \operatorname{rk} A_{j}-p r h^{0}\left(A_{j}(n)\right)\right) \\
& +r \delta \cdot \sum_{j=1}^{s} \alpha_{j} \cdot v_{j}\left(\iota_{0}\right) \cdot p \\
& +r \alpha \cdot \sum_{j=1}^{s} \alpha_{j} p \operatorname{dim} q_{t}\left(A_{j \mid N_{1}} \oplus A_{j \mid N_{2}}\right) .
\end{aligned}
$$

We divide the last expression by $p$ and observe that the assumption on the $A_{j}$ yields

$$
p \operatorname{rk} A_{j}-r h^{0}\left(A_{j}(n)\right)=\operatorname{deg}\left(A_{t}\right) \operatorname{rk} A_{j}-r \operatorname{deg}\left(A_{j}\right), \quad j=1, \ldots, s,
$$

so that the above inequality results in

$$
\begin{aligned}
0(\leq) & \sum_{j=1}^{s} \alpha_{j}\left(\operatorname{deg}\left(A_{t}\right) \mathrm{rk} A_{j}-r \operatorname{deg}\left(A_{j}\right)\right)+\delta \cdot \sum_{j=1}^{s} \alpha_{j}\left(v_{j}\left(\iota_{0}\right) \cdot r-a \cdot \operatorname{rk} A_{j}\right) \\
& +\alpha \cdot \sum_{j=1}^{s} \alpha_{j}\left(r \operatorname{dim} q_{t}\left(A_{j \mid N_{1}} \oplus A_{j \mid N_{2}}\right)-r \operatorname{rk} A_{j}\right) \\
= & \sum_{j=1}^{s} \alpha_{j}\left(\alpha-\operatorname{pardeg}\left(A_{t}\right) \operatorname{rk} A_{j}-\alpha-\operatorname{pardeg}\left(A_{j}\right) r\right) \\
& +\delta \cdot \sum_{j=1}^{s} \alpha_{j}\left(v_{j}\left(\underline{\iota}_{0}\right) \cdot r-a \cdot \operatorname{rk} A_{j}\right) .
\end{aligned}
$$

As explained on page 189 of [15],

$$
\mu_{a, b, c}\left(A^{\bullet}, \underline{\alpha} ; \varphi_{t}\right) \geq \sum_{j=1}^{s} \alpha_{j}\left(v_{j}\left(\underline{\iota}_{0}\right) \cdot r-a \cdot \operatorname{rk} A_{j}\right),
$$

so that we are done.

Proof of Theorem 6.1.4(ii). We briefly review a part of this argument in order to explain why the assumption that the stability parameter $\alpha$ be smaller than one is mandatory. For this, let $(C, 0)$ be the spectrum of a DVR $R$ with quotient field $K$. Suppose we are given a morphism $h: C \rightarrow \mathbb{G}^{\text {ss }}$ which lifts over $\operatorname{Spec}(K)$ to $\mathfrak{T}$. This lifting is given by a family $\left(\mathfrak{q}_{K}: U \otimes \pi_{\widetilde{X}}^{\star} \mathscr{O}_{\widetilde{X}}(-n) \rightarrow A_{K}, q_{K}, \varphi_{K}\right)$ over $\operatorname{Spec}(K) \times \widetilde{X}$ (we left out $\mathfrak{N}_{K}$, because it is 
trivial). This can be extended to a certain family $\left.\widetilde{\mathfrak{q}}_{C}: U \otimes \pi_{\widetilde{X}}^{\star} \mathscr{O}_{\widetilde{X}}(-n) \rightarrow \widetilde{A}_{C}, q_{C}, \varphi_{C}\right)$, consisting of

- a surjection $\widetilde{\mathfrak{q}}_{C}$ onto the flat family $\widetilde{A}_{C}$, where $\widetilde{A}_{C \mid\{0\} \times X}$ may have torsion,

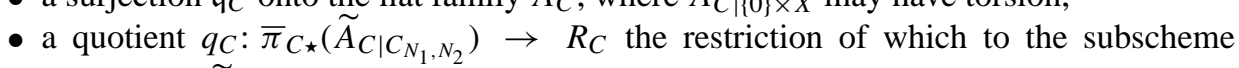
$\operatorname{Spec}(K) \times \widetilde{X}$ differs from $q_{K}$ by an element in $K^{\star}$,

- a homomorphism $\varphi_{C}:\left(\widetilde{A}_{C}^{\otimes a}\right)^{\oplus b} \rightarrow \operatorname{det}(\widetilde{A})^{\otimes c} \otimes \pi_{\widetilde{X}}^{\star} L$ the restriction of which to $\{0\} \times \widetilde{X}$ is non-trivial and the restriction of which to $\operatorname{Spec}(K) \times \widetilde{X}$ differs from $\varphi_{K}$ by an element in $K^{\star}$.

The resulting datum $k_{C}: U_{a, b} \rightarrow \pi_{C \star}\left(\operatorname{det}\left(\widetilde{A}_{C}\right)^{\otimes c} \otimes \pi_{\widetilde{X}}^{\star} L \otimes \pi_{\widetilde{X}}^{\star} \mathscr{O}_{\widetilde{X}}(n a)\right)$ defines a morphism $C \rightarrow \mathbb{G}_{2}$ which coincides with the second component $h_{2}$ of $h$, and $q_{C}$ yields a morphism $C \rightarrow \mathfrak{G}$ which coincides with the third component $h_{3}$ of $h$.

Set $A_{C}:=\widetilde{A}_{C}^{\vee v}$. This is a locally free sheaf on the smooth surface $C \times \tilde{X}$. Therefore, we have a family

$$
\mathfrak{q}_{C}: U \otimes \pi_{\tilde{X}}^{\star} \mathscr{O}_{\widetilde{X}}(-n) \rightarrow A_{C}
$$

where the kernel of the homomorphism $U \otimes \mathscr{O}_{\widetilde{X}}(-n) \rightarrow A_{C \mid\{0\} \times \widetilde{X}}$ is isomorphic to the torsion subsheaf $\mathscr{T}$ of $\widetilde{A}_{C \mid\{0\} \times X}$. Define $T:=\operatorname{dim}_{\mathbb{C}}(\mathscr{T})$. One gets a homomorphism $\bigwedge^{r} U \otimes \mathscr{O}_{C} \rightarrow \pi_{C \star}\left(\operatorname{det}\left(\widetilde{A}_{C}\right) \otimes \pi_{\widetilde{X}}^{\star} \mathscr{O}_{\widetilde{X}}(r n)\right)$ which defines a morphism $C \rightarrow \mathbb{G}_{1}$ which coincides with the first component $h_{1}$ of $h$.

Setting $A_{0}:=A_{C \mid\{0\} \times \widetilde{X}}$, we have to show that $H^{0}\left(\mathfrak{q}_{C \mid\{0\} \times \widetilde{X}}(n)\right): U \rightarrow H^{0}\left(A_{0}(n)\right)$ must be injective. This implies, in particular, that $\widetilde{A}_{C \mid\{0\} \times \widetilde{X}}$ is torsion free and, hence, $A_{C}=\widetilde{A}_{C}$ and $\mathfrak{q}_{C}=\widetilde{\mathfrak{q}}_{C}$. The family $\left(\mathfrak{q}_{C}, q_{C}, \varphi_{C}\right)$ extends the lifting of $h$ from $\operatorname{Spec}(K)$ to the whole $C$. With $H:=\operatorname{ker}\left(H^{0}\left(\mathfrak{q}_{C \mid\{0\} \times \widetilde{X}}(n)\right)\right)$ we obtain the weighted flag $(0 \subsetneq H \subsetneq$ $U,(1))$ in $U$, and we choose a one-parameter subgroup $\lambda: \mathbb{C}^{\star} \rightarrow \operatorname{SL}(U)$ which gives this weighted flag. Note that $\operatorname{dim}(H)=T$. For this special one-parameter subgroup, we find (compare [15], p. 192f])

$$
\begin{aligned}
\mu_{\mathbb{G}}(\lambda, t) & =(p-a \delta-r \alpha) \cdot \mu_{\mathbb{G}_{1}}\left(\lambda, t_{1}\right)+r \delta \cdot \mu_{\mathbb{G}_{2}}\left(\lambda, t_{2}\right)+r \alpha \cdot \mu_{\mathfrak{G}}\left(\lambda, t_{3}\right) \\
& =-(p-a \delta-r \alpha) r T-r \delta a T+r \alpha\left(p \operatorname{dim} q\left(\mathscr{T}_{N_{1}} \oplus \mathscr{T}_{N_{2}}\right)-r T\right) \\
& =-p r T+\alpha p r \operatorname{dim} q\left(\mathscr{T}_{N_{1}} \oplus \mathscr{T}_{N_{2}}\right) \\
& \leq-p r T+\alpha p r T \stackrel{\alpha<1}{<} 0 .
\end{aligned}
$$

This contradicts the fact that $h(0)$ is semistable.

\subsection{Proof of Theorem 4.2.4}

We fix an integer $s \gg 0$ as in Section 4.1 Let $(A, q, \widetilde{\tau})$ be a singular $G$-bundle with a GPS on the curve $\widetilde{X}$ and let

$$
\widehat{\varphi}:\left((A \otimes V)^{\otimes s !}\right)^{\oplus N} \rightarrow \mathscr{O}_{\widetilde{X}}
$$

be the associated homogeneous decoration. Note that, by Lemma 1.8(i) from [15], one has

$$
\frac{1}{s !} \cdot \mu(0 \subsetneq B \subsetneq A,(1) ; \widehat{\varphi}) \leq r-1
$$


for $B$ a non-trivial proper subbundle of $A$. Thus, the arguments used in the proof of Proposition 6.1.1 show that, for every subbundle $0 \subsetneq B \subsetneq A$ of $A$,

$$
\mu(B) \leq \mu(A)+\delta \cdot \frac{r-1}{r}+r \stackrel{\operatorname{deg}(A)=0}{=} \delta \cdot \frac{r-1}{r}+r,
$$

provided $(A, q, \widetilde{\tau})$ is $(\alpha, \delta)$-semistable.

The parameter space for singular $G$-bundles with a GPS. Therefore, we may choose an integer $n_{0}$ such that, for every $n \geq n_{0}$ and every $(\alpha, \delta)$-semistable singular $G$-bundle $(A, q, \widetilde{\tau})$ with a GPS, $A(n)$ is globally generated and $H^{1}(A(n))=0$. Furthermore, writing $\left(\left(\mathbb{C}^{r} \otimes V\right)^{\otimes s !}\right)^{\oplus N}$ as $\left(\left(\mathbb{C}^{r}\right)^{\otimes s !}\right)^{\oplus b}$, we may assume that $n$ is also so large that all the

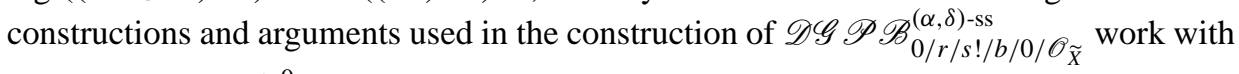
this $n$. Let $U$ and $\mathfrak{Q}^{0}$ be as in Section 6.1.

We continue with

$$
\overline{\mathfrak{Y}}=\mathfrak{Q}^{0} \times \bigoplus_{i=1}^{s} \operatorname{Hom}\left(\operatorname{Sym}^{i}(U \otimes V), H^{0}\left(\mathscr{O}_{\widetilde{X}}(i n)\right)\right) .
$$

Note that, over $\overline{\mathfrak{Y}} \times \tilde{X}$, there are universal homomorphisms

$$
\widetilde{\varphi}^{i}: \operatorname{Sym}^{i}(U \otimes V) \otimes \mathscr{O}_{\overline{\mathfrak{Y}} \times \widetilde{X}} \rightarrow H^{0}\left(\mathscr{O}_{\widetilde{X}}(i n)\right) \otimes \mathscr{O}_{\overline{\mathfrak{Y}} \times \widetilde{X}}, \quad i=1, \ldots, s .
$$

Let $\varphi^{i}=\mathrm{ev} \circ \widetilde{\varphi}^{i}$ be the composition of $\widetilde{\varphi}^{i}$ with the evaluation map

$$
\text { ev: } H^{0}\left(\mathscr{O}_{\widetilde{X}}(i n)\right) \otimes \mathscr{O}_{\overline{\mathfrak{Y}} \times \widetilde{X}} \rightarrow \pi_{\widetilde{X}}^{\star} \mathscr{O}_{\widetilde{X}}(i n), \quad i=1, \ldots, s .
$$

We twist $\varphi^{i}$ by $\operatorname{id}_{\pi_{\tilde{X}}^{\star} \mathscr{C}_{\widetilde{X}}(-i n)}$ and put the resulting maps together to get the homomorphism

$$
\varphi: \mathscr{V}_{\overline{\mathfrak{Y}}}:=\bigoplus_{i=1}^{s} \operatorname{Sym}^{i}\left(U \otimes \pi_{\widetilde{X}}^{\star} \mathscr{O}_{\widetilde{X}}(-n) \otimes V\right) \rightarrow \mathscr{O}_{\overline{\mathfrak{Y}} \times \widetilde{X}} .
$$

Next, $\varphi$ yields a homomorphism of $\mathscr{O}_{\overline{\mathfrak{Y}} \times \tilde{X}^{- \text {algebras }}}$

$$
\tilde{\tau}_{\overline{\mathfrak{Y}}}: \operatorname{Sym}^{\star}\left(\mathcal{Y}_{\overline{\mathfrak{Y}}}\right) \rightarrow \mathscr{O}_{\overline{\mathfrak{Y}} \times \tilde{X}} .
$$

On the other hand, there is a surjective homomorphism

$$
\beta: \operatorname{Sym}^{\star}(\mathscr{V} / \bar{Y}) \rightarrow \operatorname{Sym}^{\star}\left(\pi^{\star} A_{\mathfrak{Q}^{0}} \otimes V\right)^{G}
$$

of graded algebras, where the left hand algebra is graded by assigning the weight $i$ to the elements in $\operatorname{Sym}^{i}\left(U \otimes \pi_{\widetilde{X}}^{\star} \mathscr{O} \widetilde{X}(-n) \otimes V\right)$. Here, $\pi: \overline{\mathfrak{Y}} \times \widetilde{X} \rightarrow \mathfrak{Q}^{0} \times \widetilde{X}$ is the natural projection. The space $\mathfrak{Y}(G)$ is defined by the condition that $\widetilde{\tau}_{\overline{\mathfrak{Y}}}$ factorize over $\beta$, i.e., setting $A_{\mathfrak{Y}(G)}:=\left(\pi^{\star} A_{\left.\mathfrak{Q}^{0}\right)}\right)_{\mathfrak{Y}(G) \times X}$, there be a homomorphism

$$
\tilde{\tau}_{\mathfrak{Y}(G)}: \operatorname{Sym}^{\star}\left(A_{\mathfrak{Y}(G)} \otimes V\right)^{G} \rightarrow \mathscr{O}_{\mathfrak{Y}(G) \times X}
$$


with $\tilde{\tau}_{\overline{\mathfrak{Y} \mid \mathfrak{Y}(G) \times X}}=\tilde{\tau}_{\mathfrak{Y}(G)} \circ \beta$. According to Proposition 2.2.1 $\mathfrak{Y}(G)$ is defined as the scheme-theoretic intersection of the closed subschemes

$$
\mathfrak{Y}^{d}:=\left\{y \in \overline{\mathfrak{Y}} \mid \tilde{\tau}_{\overline{\mathfrak{Y}} \mid\{y\} \times X}^{d}: \operatorname{ker} \beta_{\mid\{y\} \times X}^{d} \rightarrow \mathscr{O}_{X} \text { is trivial }\right\}, \quad d \geq 0 .
$$

We find the family $\left(A_{\mathfrak{Y}(G)}, \widetilde{\tau}_{\mathfrak{Y}(G)}\right)$ on $\mathfrak{Y}(G) \times \tilde{X}$. As in the construction of the parameter space for decorated generalized parabolic bundles, we let $\mathfrak{G}$ be the Graßmannian of $r$ dimensional quotients of $U$ and $q_{\mathfrak{G}}: U \otimes \mathscr{O}_{\mathfrak{G}} \rightarrow R_{\mathfrak{G}}$ the universal quotient. We pull back the universal quotient to $\mathfrak{Y}(G) \times \mathfrak{G}$ in order to get

$$
q_{\mathfrak{Y}(G) \times \mathfrak{G}}: U \otimes \mathscr{O}_{\mathfrak{Y}(G) \times \mathfrak{G}} \rightarrow R_{\mathfrak{Y}(G) \times \mathfrak{G}} .
$$

We define $\mathfrak{q}_{\mathfrak{Y}(G) \times \mathfrak{G}}, A_{\mathfrak{Y}(G) \times \mathfrak{G}}$, and $\tilde{\tau}_{\mathfrak{Y}(G) \times \mathfrak{G}}$ as the pullbacks to $\mathfrak{Y}(G) \times \mathfrak{G} \times \tilde{X}$ of the respective objects on $\mathfrak{Y}(G) \times \tilde{X}$. Again, we have, on the scheme $\mathfrak{Y}(G) \times \mathfrak{G}=\mathfrak{Y}(G) \times$ $\mathfrak{G} \times\{N\}$, the quotient

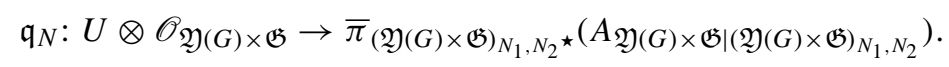

Finally, we define the closed subscheme $\mathfrak{W}(G)$ by the condition that $q_{\mathfrak{Y}(G) \times \mathfrak{G}}$ vanish on $\operatorname{ker}\left(\mathfrak{q}_{N}\right)$. The restriction of $q_{\mathfrak{Y}(G) \times \mathfrak{G}}$ to $\mathfrak{W}(G)$ factorizes over a quotient

$$
q_{\mathfrak{W}(G)}: \bar{\pi}_{\mathfrak{W}(G)_{N_{1}, N_{2} \star}}\left(A_{\mathfrak{W}(G) \mid \mathfrak{W}(G)_{N_{1}, N_{2}}}\right) \rightarrow R_{\mathfrak{W}(G)}:=R_{\mathfrak{Y}(G) \times \mathfrak{G} \mid \mathfrak{W}(G) .}
$$

The restrictions $A_{\mathfrak{W}(G)}$ of $A_{\mathfrak{Y}(G) \times \mathfrak{G}}$ and $\tilde{\tau}_{\mathfrak{W}(G)}$ of $\tilde{\tau}_{\mathfrak{Y}(G) \times \mathfrak{G}}$ to $\mathfrak{W}(G) \times \tilde{X}$ and $q_{\mathfrak{W}(G)}$ provide us with the universal family $\left(A_{\mathfrak{W}(G)}, q_{\mathfrak{W}(G)}, \widetilde{\tau}_{\mathfrak{W}(G)}\right)$. Of course, the analog of Proposition 6.1.2 holds true.

The group action. This time, we find on our parameter space $\mathfrak{W}(G)$ an action

$$
\Gamma^{\prime}: \operatorname{GL}(U) \times \mathfrak{W}(G) \rightarrow \mathfrak{W}(G)
$$

of the group GL(U). Again, the property analogous to the one of Proposition 6.1.3 is fulfilled. For the purpose of taking the GIT quotient, we may regard this action as an action

$$
\widetilde{\Gamma}:\left(\mathbb{C}^{\star} \times \operatorname{SL}(U)\right) \times \mathfrak{W}(G) \rightarrow \mathfrak{W}(G)
$$

of the group $\mathbb{C}^{\star} \times \operatorname{SL}(U)$. The quotient may then be taken in two steps. First, we form $\overline{\mathfrak{W}}(G):=\mathfrak{W}(G) / / \mathbb{C}^{\star}$, which poses no problems, and then $\overline{\mathfrak{W}}(G) / / \operatorname{SL}(U)$. The latter problem will be reduced to the case of decorated generalized parabolic bundles. To this end, we note that the construction from Section 4.2 can clearly also be performed in families, that is, the homomorphism $\tilde{\tau}_{\mathfrak{W}(G)}$ induces the homomorphism

$$
\widehat{\varphi}_{\mathfrak{W}(G)}:\left(\left(A_{\mathfrak{W}(G)} \otimes V\right)^{\otimes s !}\right)^{\oplus N} \rightarrow \mathscr{O}_{\mathfrak{W}(G) \times \widetilde{X}}
$$

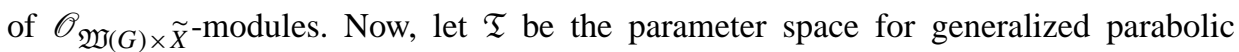
bundles $(A, q, \varphi)$ with $\operatorname{deg}(A)=0$ and $\operatorname{rk}(A)=r$ and a decoration of type $\left(s !, b, 0 ; \mathscr{O}_{\widetilde{X}}\right)$. 
By the local universal property of $\mathfrak{T}$ (Proposition 6.1.2), the triple $\left(q_{\mathfrak{W}(G)}: U \otimes \pi_{\widetilde{X}}^{\star} \mathscr{O}_{\widetilde{X}}(-n)\right.$ $\left.\rightarrow A_{\mathfrak{W}(G)}, q_{\mathfrak{W}(G)}, \widehat{\varphi}_{\mathfrak{W}(G)}\right)$ induces a morphism

$$
\Phi: \mathfrak{W}(G) \rightarrow \mathfrak{T} .
$$

If we let $\mathbb{C}^{\star}$ act trivially on $\mathfrak{T}$, then $\Phi$ becomes $\left(\mathbb{C}^{\star} \times \operatorname{SL}(U)\right)$-equivariant. It thus descends to an $\operatorname{SL}(U)$-equivariant morphism

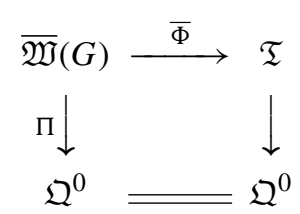

over the quot scheme $\mathfrak{Q}^{0}$. Since $\Pi$ is proper by the construction of $\mathfrak{W}(G)$ and $\bar{\Phi}$ is injective, we deduce

Lemma 6.2.1. The morphism $\overline{\Phi:} \overline{\mathfrak{W}}(G) \rightarrow \mathfrak{T}$ is finite.

Set

$$
\overline{\mathfrak{W}}(G)^{(\alpha, \delta)-(\mathrm{s}) \mathrm{s}}:=\bar{\Phi}^{-1}\left(\mathfrak{T}^{(\alpha, \delta)-(\mathrm{s}) \mathrm{s}}\right) \quad \text { and } \quad \mathfrak{W}(G)^{(\alpha, \delta)-(\mathrm{s}) \mathrm{s}}:=\Phi^{-1}\left(\mathfrak{T}^{(\alpha, \delta)-(\mathrm{s}) \mathrm{s}}\right) .
$$

Then the map

$$
\bar{\Phi}_{\mid \overline{\mathfrak{W}}(G)^{(\alpha, \delta)-(\mathrm{s}) \mathrm{s}}}: \overline{\mathfrak{W}}(G)^{(\alpha, \delta)-(\mathrm{s}) \mathrm{s}} \rightarrow \mathfrak{T}^{(\alpha, \delta)-(\mathrm{s}) \mathrm{s}}
$$

is also proper, so that the quotients

$$
\begin{aligned}
\mathscr{S}^{(\mathrm{s}) \mathrm{s}}:=\mathscr{S} \mathscr{P} \mathscr{B} \mathscr{G} \mathscr{P} \mathscr{S}(\varrho)^{(\alpha, \delta)-(\mathrm{s}) \mathrm{s}}: & =\overline{\mathfrak{W}}(G)^{(\alpha, \delta)-(\mathrm{s}) \mathrm{s}} / / \mathrm{SL}(U) \\
& =\mathfrak{W}(G)^{(\alpha, \delta)-(\mathrm{s}) \mathrm{s}} / /\left(\mathbb{C}^{\star} \times \operatorname{SL}(U)\right)
\end{aligned}
$$

exist, the scheme $\mathscr{S}^{\text {ss }}$ being projective. By our definition of $(\alpha, \delta)$-(semi)stability, the open subsets $\mathfrak{W}(G)^{(\alpha, \delta)-(s) \mathrm{s}}$ consist precisely of those points $t$ for which the restriction $\left(A_{t}, q_{t}, \widetilde{\tau}_{t}\right)$ of the universal family to $\{t\} \times \widetilde{X}$ is $(\alpha, \delta)$-(semi)stable. Therefore, Theorem 4.2.4 is deduced as before.

\subsection{Proof of the Main Theorem}

Given $\alpha$, we choose $\delta>\delta_{\infty}$ in accordance with Proposition 4.2 .3 Thus, a descending $G$ bundle $(A, q, \widetilde{\tau})$ on $\widetilde{X}$ is $\alpha$-(semi)stable if and only if it is an $(\alpha, \delta)$-(semi)stable singular $G$-bundle with a GPS.

Let $\mathfrak{W}(G)$ be as above, carrying the universal family $\left(A_{\mathfrak{W}(G)}, q_{\mathfrak{W}(G)}, \widetilde{\tau}_{\mathfrak{W}(G)}\right)$. As explained in Section 4.3 , we have an associated homomorphism

$$
\tau_{\mathfrak{W}(G)}^{\prime}: \operatorname{Sym}^{\star}\left(\mathscr{A}_{\mathfrak{W}(G)} \otimes V\right)^{G} \rightarrow\left(\operatorname{id}_{\mathfrak{W}(G)} \times \nu\right)_{\star} \mathscr{O}_{\mathfrak{W}(G) \times \widetilde{X}}
$$


of $\mathscr{O}_{\mathfrak{W}(G) \times X}$-algebras. Invoking Proposition 2.2.1 once more, we define $\mathfrak{D}(G)$ as the closed subscheme of $\mathfrak{W}(G)$ where the induced homomorphism

$\operatorname{Sym}^{\star}\left(\mathscr{A}_{\mathfrak{W}(G)} \otimes V\right)^{G} \rightarrow\left(\left(\operatorname{id}_{\mathfrak{W}(G)} \times v\right)_{\star} \mathscr{O}_{\mathfrak{W}(G) \times \tilde{X}}\right) / \mathscr{O}_{\mathfrak{W}(G) \times X \stackrel{2.1 .1}{\cong} \pi_{X}^{2.1 .2}}^{\cong} \pi_{X}^{\star}\left(\left(v_{\star} \mathscr{O}_{\tilde{X}}\right) / \mathscr{O}_{X}\right)$

vanishes. Now, suppose we are given a family $\left(A_{S}, q_{S}, \tilde{\tau}_{S}\right)$ of $(\alpha, \delta)$-semistable singular $G$-bundles with a GPS parameterized by the scheme $S$. Choose a covering $S_{i}, i \in I$, of $S$ such that the above family is induced on each $S_{i}$ by a morphism $f_{i}: S_{i} \rightarrow \mathfrak{W}(G), i \in I$. By the universal property of $\mathfrak{D}(G)$ (Proposition 2.2.1) and Lemma 4.3.1, the morphisms $f_{i}$ factorize over $\mathfrak{D}(G)$ for all $i \in I$ if and only if $\left(A_{S}, q_{S}, \tilde{\tau}_{S}\right)$ is a family of $(\alpha, \delta)$ semistable descending $G$-bundles. On the other hand, our constructions imply that the quotients

$$
\mathscr{M}(\varrho)^{\alpha-(\mathrm{s}) \mathrm{s}}:=\left(\mathfrak{D}(G) \cap \mathfrak{W}(G)^{(\alpha, \delta)-(\mathrm{s}) \mathrm{s}}\right) / /\left(\mathbb{C}^{\star} \times \operatorname{SL}(U)\right)
$$

exist and that $\mathscr{M}(\varrho)^{\alpha-s s}$ is projective. It is clear that the spaces $\mathscr{M}(\varrho)^{\alpha-s s}$ and $\mathscr{M}(\varrho)^{\alpha-s}$ are the moduli spaces we have been looking for.

Acknowledgements. The author acknowledges support by the DFG through a Heisenberg fellowship and through the priority program "Globale Methoden in der komplexen Geometrie - Global Methods in Complex Geometry".

Substantial parts of the paper were conceived during the author's visit to the Tata Institute of Fundamental Research, Mumbai, the Institute of Mathematical Sciences, Chennai, and the Chennai Mathematical Institute. The author would like to thank Professor Bhosle, Professor Nagaraj, and Professor Seshadri for the respective invitations and several interesting discussions. Several passages were written down during the author's visit to the Consejo Superior de Investigaciones Científicas (CSIC) in Madrid which was funded by the European Differential Geometry Endeavour (EDGE), EC FP5 contract no. HPRN-CT-2000-00101. The author wishes to thank O. García-Prada for the invitation and hospitality.

I thank the referee for the references [5] and [8].

\section{References}

[1] Balaji, V., Seshadri, C. S.: Semistable principal bundles. I. Characteristic zero. Special issue in celebration of Claudio Procesi's 60th birthday, J. Algebra 258, 321-347 (2002) Zbl pre01868091 MR 1958909

[2] Bhosle, U.: Generalised parabolic bundles and applications to torsionfree sheaves on nodal curves. Ark. Mat. 30, 187-215 (1992) Zbl 0773.14006 MR 1289750

[3] Bhosle, U.: Tensor fields and singular principal bundles. Int. Math. Res. Not. 2004, No. 57 , 3057-3077

[4] Borel, A.: Linear Algebraic Groups. 2nd ed., Grad. Texts in Math. 126, Springer, New York (1991) Zbl 0726.20030 MR 1102012

[5] Burban, I., Drozd, Y.: Coherent sheaves on rational curves with simple double points and transversal intersections. Duke Math. J. 121, 189-229 (2004) MR 2034641

[6] Faltings, G.: Moduli-stacks for bundles on semistable curves. Math. Ann. 304, 489-515 (1996) Zbl 0847.14018 MR 1375622

[7] Friedman, R., Morgan, J. W.: Holomorphic principal bundles over elliptic curves. II. The parabolic construction. J. Differential Geom. 56, 301-379 (2000) Zbl 1033.14016 MR 1863019 
[8] Friedman, R., Morgan, J. W.: Holomorphic principal bundles over elliptic curves. III. Singular curves and fibrations. math.AG/0108104, 76 pages

[9] Gómez, T. L., Sols, I.: Stable tensors and moduli space of orthogonal sheaves. math.AG/0103150, 36 pp.

[10] Gómez, T. L., Sols, I.: Moduli space of principal sheaves over projective varieties. Ann. of Math. (to appear), see also math.AG/0206277, 48 pp.

[11] Grothendieck, A.: Éléments de géométrie algébrique II. Étude globale élémentaire de quelques classes de morphismes. Inst. Hautes Études Sci. Publ. Math. 8, 222 pp. (1961) Zbl 0118.36206 MR 0217084

[12] Mumford, D. et al.: Geometric Invariant Theory. 3rd ed., Ergeb. Math. Grenzgeb. (2) 34, Springer, Berlin (1994) Zbl 0797.14004 MR 1304906

[13] Nagaraj, D. S., Seshadri, C. S.: Degenerations of the moduli spaces of vector bundles on curves I, Proc. Indian Acad. Sci. Math. Sci. 107, 101-137 (1997) Zbl 0922.14023 MR 1455315

[14] Ramanathan, A.: Moduli for principal bundles over algebraic curves I-II, Proc. Indian Acad. Sci. Math. Sci. 106, 301-28, 421-449 (1996) Zbl 0901.14008 MR 1420170

[15] Schmitt, A. H. W.: A universal construction for moduli spaces of decorated vector bundles over curves. Transformation Groups 9, 167-209 (2004) MR 2056535

[16] Schmitt, A. H. W.: Singular principal bundles over higher-dimensional manifolds and their moduli spaces. Int. Math. Res. Not. 2002, No. 23, 1183-1209 Zbl 1034.14017 MR 1903952

[17] Schmitt, A. H. W.: A closer look at semistability for singular principal bundles. Int. Math. Res. Not. 2004, No. 62, 3327-3366 MR 2097106

[18] Schmitt, A. H. W.: Global boundedness for decorated sheaves. Int. Math. Res. Not. 2004, No. $68,3637-3671$

[19] Serre, J.-P.: Espaces fibrés algébriques. In: Séminaire Chevalley, 1958, also Documents Mathématiques 1, 107-140 (2001)

[20] Springer, T. A.: Linear Algebraic Groups. 2nd ed., Progr. Math. 9, Birkhäuser Boston, Boston, MA (1998) Zbl 0927.20024 MR 1642713

[21] Sun, X.: Degeneration of moduli spaces and generalized theta functions. J. Algebraic Geom. 9, 459-527 (2000) Zbl 0971.14030 MR 1752012

[22] Sun, X.: Moduli spaces of SL(r)-bundles on singular irreducible curves. Asian J. Math. 7, 609-626 (2003) MR 2074894 